\title{
EXISTENCE OF CONVEX HYPERSURFACES WITH PRESCRIBED GAUSS-KRONECKER CURVATURE
}

\author{
XU-JIA WANG
}

\begin{abstract}
Let $f(x)$ be a given positive function in $R^{n+1}$. In this paper we consider the existence of convex, closed hypersurfaces $X$ so that its GaussKronecker curvature at $x \in X$ is equal to $f(x)$. This problem has variational structure and the existence of stable solutions has been discussed by Tso (J. Diff. Geom. 34 (1991), 389-410). Using the Mountain Pass Lemma and the Gauss curvature flow we prove the existence of unstable solutions to the problem.
\end{abstract}

\section{INTRODUCTION}

In this paper we are concerned with the existence of a convex, closed hypersurface $X$ so that its Gauss-Kronecker curvature $K$ is equal to $f$, i.e.,

$$
K(p)=f(p) \quad \forall p \in X,
$$

where $f(x)$ is a $C^{2}$ smooth, positive and locally bounded function on $\mathbb{R}^{n+1}$.

Such kind of problems was raised by Yau [13], in which he proposed to study the existence of hypersurfaces in $\mathbb{R}^{n+1}$ so that their mean curvature is equal to a given function. Equation (1.1) has been investigated by Oliker [6] in which he proved that if there exist $R_{1}$ and $R_{2}, 0<R_{1}<R_{2}$, so that

$$
f(x)>R_{1}^{-n} \quad \text { for } \quad|x|=R_{1} \text { and } f(x)<R_{2}^{-n} \text { for }|x|=R_{2},
$$

and $\frac{\partial}{\partial \rho} \rho^{n} f(\rho x) \leq 0$ for $x \in S^{n}, \rho>0$, then there exists a solution to the equation (1.1). It was subsequently proved by Delanoë [5] that (1.2) alone is sufficient for the existence.

Equation (1.1) was later studied by Tso [10] via variational approach. Let $\sigma_{k}$ $(k=0,1, \cdots, n+1)$ denote the $k$ th normalized elementary symmetric function of the principal curvatures of a convex hypersurface $X$, namely, $\sigma_{k}=\sum_{i_{1}<\cdots<i_{k}} k_{i_{1}} \cdots$ $k_{i_{k}} / C_{n}^{k}\left(\operatorname{set} \sigma_{0}=1, \sigma_{n+1}=0\right)$. Let $I_{k}(X)=\frac{1}{n-k} \int_{X} \sigma_{k}$. It is known [9] that $I_{k}$ satisfies the variational formula

$$
\left\langle\delta I_{k}(X), \xi\right\rangle=\int_{X} \sigma_{k+1}\langle\xi, \gamma\rangle d \sigma, \quad k=0,1, \cdots, n
$$

Received by the editors April 3, 1995 and, in revised form, July 5, 1995.

1991 Mathematics Subject Classification. Primary 53C45, 58G11, 35J60.

Key words and phrases. Gauss curvature, convex hypersurface, existence.

This work was finished while the author was visiting the Mathematical Section of the International Center for Theoretical Physics. He would like to thank the center for its support.

(C)1996 American Mathematical Society 
where $\xi$ is any smooth vector field on $X, \gamma$ is the unit outward normal of $X$. By $\langle x, y\rangle$ we denote the inner product in Euclidean space. Let

$$
J(X)=I_{n-1}-\int_{C l(X)} f(x) d x
$$

where $C l(X)$ denotes the convex body enclosed by $X$. Then

$$
\langle\delta J(X), \xi\rangle=\int_{X}\left(\sigma_{n}-f\right)\langle\xi, \gamma\rangle d \sigma
$$

Consequently to find a solution of (1.1) is formally equivalent to looking for a critical point of the functional $J(X)$.

Let $\chi$ denote the set of all convex $C^{1,1}$ hypersurfaces with the topology induced by the distance

$$
\operatorname{dist}\left(X_{1}, X_{2}\right)=\sup _{p_{1} \in X_{1}} \inf _{p_{2} \in X_{2}}\left|p_{1}-p_{2}\right| .
$$

A solution $X$ is said to be stable if it is a local minimizer of $J$, namely, there exists an open subset $\chi_{0} \subset \chi$ which contains $X$ so that $J(X)=\inf \left\{J(\widetilde{X}), \widetilde{X} \in \chi_{0}\right\}$ and $J(X)<J(\widetilde{X}) \forall \widetilde{X} \in \partial \chi_{0}$. Otherwise it is said to be unstable. The condition (1.2) above implies $J$ has a local minimum. In [10] Tso proved that if $f$ is integrable over $\mathbb{R}^{n+1}$ and there exists a convex hypersurface $X_{0}$ so that $J\left(X_{0}\right) \leq 0$, then (1.1) admits a stable solution which reaches the absolute minimum of $J$. Using the Mountain Pass Lemma, in this paper we will prove

Theorem 1. If (1.1) admits a stable solution $X$, then it has an unstable solution contained inside $X$.

From Theorem 1 we see that (1.1) admits at least two solutions if $f$ satisfies (1.2), or if $f$ is integrable over $\mathbb{R}^{n+1}$ and there exists $X_{0}$ so that $J\left(X_{0}\right) \leq 0$. In Theorem 3.2 we will prove that if (1.1) has a stable solution $X$ and if $f(x)$ satisfies

$$
\lambda \leq f(x) \leq \Lambda \quad \forall x \in \mathbb{R}^{n+1},
$$

for some positive constants $\lambda$ and $\Lambda$, then (1.1) has an unstable solution $X_{1}$ such that $X$ lies inside $X_{1}$.

But equation (1.1) usually admits no stable solution. It is easy to see that the unit sphere $S^{n}$ is the unique unstable solution of (1.1) if $f \equiv 1$. Hence it is interesting to find unstable solutions of (1.1) without the assumption that (1.1) has a stable solution. A natural question is to ask whether there is a solution to (1.1) if $f(x)$ satisfies (1.5). In this case $J(X)$ is neither bounded from above nor from below. The difficulty in finding unstable solutions is that $J$ lacks compactness, which is a common feature for many prescribed curvature problems, such as the Yamabe problem and the problem of finding surfaces with constant mean curvature. The basic idea in treating the latter two problems consists in finding a Palais-Smale sequence. The problem (1.1) is somewhat different, for $J(X)$ is defined only on a topology space. The existence of solutions of (1.1) turns out to be strongly dependent on the behaviour of $f$. Let $f(x)=3+\operatorname{arctg} x_{1}$, we will show from the necessary condition for the Minkowski problem that there is no solution to equation (1.1). Our purpose here is to find some reasonable conditions on $f(x)$ so that (1.1) admits a solution. In particular we will prove

Theorem 2. Suppose $\lim _{|x| \rightarrow \infty} f(x)=f_{0}>0$. Then (1.1) has an unstable solution. 
The proof of Theorems 1 and 2 is based on the study of the logarithmic gradient flow

$$
\frac{\partial}{\partial t} X(x, t)=-\log (K / f(X)) \cdot \gamma,
$$

where $\gamma$ is the unit outward normal of $X$ at $X(x, t), K$ denotes the Gauss curvature of $X$ at $X(x, t)$, and $X$ is parametrized on $S^{n}$. Let $X(\cdot, t)$ be a solution of (1.6). From (1.4) we have

$$
\frac{d}{d t} J(X(\cdot, t))=-\int_{X}(K-f) \log (K / f) d \sigma \leq 0 .
$$

We will establish in $\S 2$ the a priori estimates for solutions of (1.1) and (1.6). In $\S 3$ and $\S 4$ we prove Theorems 1 and 2, respectively. The following comparison principle will be used repeatedly.

Comparison Principle. Suppose $X_{i}(\cdot, t),(i=1,2)$ are smooth solutions of

$$
\frac{\partial}{\partial t} X=-\log \left(K / f_{i}\right) \cdot \gamma
$$

with initial conditions $X_{i}(\cdot, 0)=X_{0, i}$. If $f_{1} \leq f_{2}$ and $X_{0,1} \subset C l\left(X_{0,2}\right)$, then $X_{1}(\cdot, t) \subset C l\left(X_{2}(\cdot, t)\right)$ for $t>0$. If moreover $X_{0,1} \neq X_{0,2}$, then $X_{1}(\cdot, t)$ is strictly contained in $X_{2}(\cdot, t)$ for $t>0$.

Notation. By convex hypersurface we mean a strictly convex $C^{1,1}$ hypersurface, except otherwise specified. For a convex hypersurface $X$, we denote by $\operatorname{mes}(X)=$ $\int_{X} d \sigma$ the area of $X$, by $\operatorname{Vol}(X)=\frac{1}{n+1} \int_{X} H d \sigma$ the volume enclosed by $X$, where $H$ is the support function of $X$. The length and width of $X$, denoted by $R(X)$ and $r(X)$, respectively, are defined by

$$
\begin{aligned}
& R(X)=\sup _{x \in S^{n}}[H(x)+H(-x)], \\
& r(X)=\inf _{x \in S^{n}}[H(x)+H(-x)] .
\end{aligned}
$$

The support center of $X$ is defined in this paper by

$$
p(X)=\int_{S^{n}} H(x) x d \sigma .
$$

By $C l(X)$ we denote the closure of $X$, which is a convex body in $\mathbb{R}^{n+1}$. For any two convex hypersurfaces $X_{1}$ and $X_{2}$, we will write for convenience $X_{1} \leq X_{2}$ if $X_{1} \subset C l\left(X_{2}\right)$, and if $X_{1}$ is strictly contained in $C l\left(X_{2}\right)$, we will write $X_{1}<X_{2}$.

\section{A priori estimates}

We first establish the a priori estimates for solutions of (1.1). To do so it is convenient to reduce the equation (1.1) to an elliptic Monge-Ampère equation for the support function of $X$.

For any convex hypersurface $X$, its support function $H$ is defined on $\mathbb{R}^{n+1}$ by

$$
H(x)=\sup \{\langle x, p\rangle ; p \in X\}, \quad x \in \mathbb{R}^{n+1} .
$$

It is convex and homogeneous of degree 1 . If $X$ is strictly convex and smooth, so is $H(x)$; and $X$ can be recovered from $H(x)$ by

$$
p(x)=\nabla H(x)=\left(H_{1}(x), \cdots, H_{n+1}(x)\right), \quad x \in S^{n},
$$


with $x$ as the unit outer normal of $X$ at $p$. Let $\left\{e_{1}, \cdots, e_{n}\right\}$ be a locally orthonormal frame field on $S^{n}$. The radii of the principal curvatures of $X$ at $p(x), x \in S^{n}$, are exactly the eigenvalues of the matrix $\left(H_{\alpha \beta}(x)+H(x) I\right)$, where $I$ denotes the unit matrix, the subscripts $\alpha, \beta$ (Greek letters) denote covariant derivatives on $S^{n}$. Thus

$$
K(p(x))=\left[\operatorname{det}\left(H_{\alpha \beta}(x)+H(x) I\right)\right]^{-1}, \quad x \in S^{n} .
$$

Hence if $X$ is a smooth solution of (1.1), its support function $H(x)$ satisfies

$$
\operatorname{det}\left(H_{\alpha \beta}+H I\right) f(\nabla H)=1 \text { on } S^{n},
$$

and vice versa.

Let $u(y), y=\left(y_{1}, \cdots, y_{n}\right)$, be the restriction of $H$ on $y_{n+1}=-1$. By $(2.4), u(y)$ satisfies

$$
\log \operatorname{det}\left(D^{2} u\right)=\widetilde{f}(y, u, D u)=: \log \left[\left(1+|y|^{2}\right)^{-(n+2) / 2} / f\left(D u, \sum_{i=1}^{n} y_{i} \frac{\partial u}{\partial y_{i}}-u\right)\right]
$$

Lemma 2.1. Let $H \in C^{4}\left(S^{n}\right)$ be a solution of (2.4) and $X$ the associated hypersurface. Then

$$
\sup _{x \in S^{n}}\left(H_{\alpha \alpha}(x)+H(x)\right) \leq \widetilde{C},
$$

where $\widetilde{C}$ depends only on $n, R(X)$, and $\log f$ up to its second derivatives.

Proof. Let $X$ be the convex hypersurface associated with $H$. By proper choice of the coordinates we may suppose $X$ contains the origin, namely, $H>0$. Note that $\left\{H_{\alpha \beta}+H I\right\}$ is invariant after subtracting a linear function.

Let

$$
\varphi(x, \alpha)=\log \left[H_{\alpha \alpha}(x)+H(x)\right]+\beta \sum_{i=1}^{n+1} H_{i}^{2},
$$

where $\beta>0$ is a constant to be determined. Suppose the $\operatorname{supremum}_{\sup _{x, \alpha}} \varphi(x, \alpha)$ is attained at the south pole and $\alpha_{0}=(1,0, \cdots, 0)$. Then $\alpha_{0}$ is a principal direction of $H$ at the south pole, hence we may suppose $\left(H_{\alpha \beta}\right)$ is diagonal at this point. Let

$$
\alpha=\alpha(x)=\left(1-x_{1}^{2},-x_{1} x_{2}, \cdots,-x_{1} x_{n+1}\right) / \sqrt{1-x_{1}^{2}} .
$$

Direct computation shows that

$$
\begin{gathered}
\sum_{i=1}^{n+1} H_{i}^{2}(x)=\sum_{i=1}^{n} u_{i}^{2}(y)+\left|u(y)-\sum_{i=1}^{n} y_{i} u_{i}(y)\right|^{2}, \\
H_{\alpha \alpha}(x)+H(x)=u_{11}(y) \cdot \frac{\left(1+|y|^{2}\right)^{3 / 2}}{1+|y|^{2}-y_{1}^{2}},
\end{gathered}
$$

where $y=-\left(x_{1}, \cdots, x_{n}\right) / x_{n+1} \in \mathbb{R}^{n}$, and $u_{i}=\partial u / \partial y_{i}$. Hence the function

$$
\psi(y)=\log u_{11}+\log \frac{\left(1+|y|^{2}\right)^{3 / 2}}{1+|y|^{2}-y_{1}^{2}}+\beta\left[\sum_{i=1}^{n} u_{i}^{2}+\left(u-\sum_{i=1}^{n} y_{i} u_{i}\right)^{2}\right]
$$

attains its maximum at $y=0$. At this point we have

$$
\psi_{k}=\frac{u_{11 k}}{u_{11}}+2 \beta u_{i} u_{i k}=0
$$


and

$$
\psi_{k k}=\frac{u_{k k 11}}{u_{11}}-\frac{u_{11 k}^{2}}{u_{11}^{2}}+\tau_{k}+2 \beta\left[u_{i k}^{2}+u_{i} u_{i k k}-u u_{k k}\right] \leq 0,
$$

where $\tau_{k}=3$ if $k=1$ and $\tau_{k}=1$ if $k>1$. Differentiating the equation (2.5) yields

$$
\begin{gathered}
u^{i j} u_{i j k}=\frac{\partial}{\partial x_{k}} \widetilde{f}, \\
u^{i j} u_{i j k k}-u^{i j} u^{r s} u_{i r k} u_{j s k}=\frac{\partial^{2}}{\partial x_{k}^{2}} \widetilde{f},
\end{gathered}
$$

where $\left\{u^{i j}\right\}$ is the inverse of $\left\{u_{i j}\right\}$. Multiplying (2.9) by $u^{k k}$ and summing, we obtain

$$
0 \geq u^{k k} \psi_{k k} \geq u^{k k}+2 \beta\left[u_{k k}+u_{i} \frac{\partial}{\partial x_{i}} \widetilde{f}-n u\right]+\frac{1}{u_{11}} \frac{\partial^{2}}{\partial x_{1}^{2}} \widetilde{f}
$$

where summing convention is used. By (2.8) we have

$$
\begin{aligned}
2 \beta u_{i} \frac{\partial}{\partial x_{i}} \widetilde{f}+\frac{1}{u_{11}} \frac{\partial^{2}}{\partial x_{1}^{2}} \widetilde{f} & =2 \beta u_{i} \widetilde{f}_{p_{i}} u_{i i}+\frac{1}{u_{11}}\left[\widetilde{f}_{p_{i}} u_{i 11}+\widetilde{f}_{p_{1} p_{1}} u_{11}^{2}\right]+O(1) \\
& =\widetilde{f}_{p_{1} p_{1}} u_{11}+O(1),
\end{aligned}
$$

where $O(1)$ depends only on $n, \beta,\|u\|_{C^{1}}$, and $\widetilde{f}$ up to its second derivatives. Letting $\beta=1+\sup \left|\nabla_{p}^{2} \widetilde{f}\right|$ and noticing that for any support function $H$,

$$
\sup \left\{|\nabla H(x)|, x \in S^{n}\right\} \leq \sup \left\{H(x), x \in S^{n}\right\},
$$

we obtain $u_{k k}+u^{k k} \leq C$. Hence (2.6) holds.

Lemma 2.1 implies that (2.4) is uniformly elliptic. By Krylov's $C^{2+\alpha}$ estimates we have

$$
\|H\|_{C^{3+\alpha}\left(S^{n}\right)} \leq C
$$

for some $C$ depending only on $n, R(X)$, and $\log f$ up to its second derivatives.

Next we consider the problem

$$
\frac{\partial}{\partial t} X(x, t)=-\log (K / f(X)) \cdot \gamma, \quad X(\cdot, 0)=X_{0},
$$

where $X_{0}$ is a $C^{4}$ smooth and strictly convex hypersurface parametrized on $S^{n}$. Equations of this type have been studied by Tso [10], Chou and the author [4]. In [4] we present a new appreach to the Minkowski problem by studying the asymptotical convergence of solutions of the associated Gauss-Kronecker curvature flow.

Let $X(\cdot, t)$ be a solution of $(2.11)$ and $H(\cdot, t)$ its support function. Then $\left\langle X_{t}, \gamma\right\rangle=$ $-H_{t}$, see [12]. By $(2.3)$ we therefore reduce $(2.11)$ to

$$
H_{t}=\log \operatorname{det}\left(H_{\alpha \beta}+H I\right)+\log f(\nabla H) \quad \text { on } S^{n} \times[0,+\infty),
$$

with initial condition $H(\cdot, 0)=H_{0}$, where $H_{0}$ is the support function of $X_{0}$. Conversely, if $H(x, t)$ is a solution of $(2.12)$, the hypersurfaces recovered from $H(x, t)$ satisfy (2.11). Let $u(x, t)=H(x,-1, t), x \in \mathbb{R}^{n}$. Then $u$ satisfies

$$
u_{t}(x, t)=\sqrt{1+|x|^{2}} \log \operatorname{det}\left(D^{2} u\right)+g(x, u, D u),
$$


where

$$
g(x, u, D u)=\frac{n+2}{2} \sqrt{1+|x|^{2}} \log \left(1+|x|^{2}\right)+\sqrt{1+|x|^{2}} \log f\left(D u, \sum_{i=1}^{n} x_{i} u_{i}-u\right) .
$$

Lemma 2.2. Let $H(x, t) \in C^{4,2}\left(S^{n} \times[0, T]\right)$ be a solution of (2.12). Then

$$
\begin{gathered}
\sup _{S^{n} \times[0, T]}\left[H_{\alpha \alpha}(x, t)+H(x, t)\right] \leq \widetilde{C}, \\
\sup _{S^{n} \times[0, T]}\left|H_{t}(x, t)\right| \leq C,
\end{gathered}
$$

where $\widetilde{C}$ depends only on $n, \sup _{[0, T]} R(X(\cdot, t)), f$, and the initial condition $H_{0}(x) ; C$ depends only on $n, f, \inf _{[0, T]} r(X(\cdot, t)), \sup _{[0, T]} R(X(\cdot, t))$ and $H_{0}(x)$. In particular, if the supremum in (2.14) (or (2.15)) is attained at $t>0$, then the constant $\widetilde{C}$ (or $C)$ is independent of the initial data $H_{0}$.

Proof. For any given $T>0$, by Lemma 2.3 in [4], one can construct a piecewise smooth function $s(t)=\left(s_{1}(t), \cdots, s_{n+1}(t)\right) \in C^{0,1}\left([0, T], \mathbb{R}^{n+1}\right)$ so that

$$
\begin{gathered}
\left|s^{\prime}(t)\right| \leq 2(n+1) \sup _{x \in S^{n}, t \in[0, T]} H_{t}(x, t), \\
\sup _{x \in S^{n}, t \in[0, T]}|H(x, t)-s(t) \cdot x| \leq 2(n+1) \sup _{t \in[0, T]} R(X(\cdot, t)) .
\end{gathered}
$$

Let

$$
\varphi=\log \left(H_{\alpha \alpha}+H\right)+\beta|\nabla H-s|^{2},
$$

where $\beta=1+\sup \left|\nabla_{p}^{2} g\right|$. Suppose the supremum $\sup \left\{\varphi,(x, t) \in S^{n} \times[0, T]\right\}$ is attained at the south pole $x=(0, \cdots, 0,-1), t=\bar{t}>0$, and $\alpha=e_{1}$, the $x_{1}$ direction. By proper choice of the coordinates we may suppose $\left(H_{\alpha \beta}\right)$ is diagonal at this point. Let $u(y, t)=H(y,-1, t)$. Similar to $(2.7)$ we see that the function

$$
\psi(y, t)=\log u_{11}+\log \frac{\left(1+|y|^{2}\right)^{3 / 2}}{1+|y|^{2}-y_{1}^{2}}+\beta\left[\sum_{i=1}^{n}\left(u_{i}-s_{i}\right)^{2}+\left|u+s_{n+1}-\sum_{i=1}^{n} y_{i} u_{i}\right|^{2}\right]
$$

attains its maximum at $(y, t)=(0, \bar{t})$. At this point we have

$$
\begin{gathered}
0 \leq \psi_{t}=\frac{u_{11 t}}{u_{11}}+2 \beta\left[\left(u_{i}-s_{i}\right)\left(u_{i t}-s_{i}^{\prime}\right)+\left(u+s_{n+1}\right)\left(u_{t}+s_{n+1}^{\prime}\right)\right], \\
0=\psi_{k}=\frac{u_{11 k}}{u_{11}}+2 \beta\left(u_{i}-s_{i}\right) u_{i k},
\end{gathered}
$$

and

$$
0 \geq \psi_{k k} \geq \frac{u_{k k 11}}{u_{11}}-\frac{u_{11 k}^{2}}{u_{11}^{2}}+1+2 \beta\left[u_{i k}^{2}+\left(u_{i}-s_{i}\right) u_{i k k}-\left(u+s_{n+1}\right) u_{k k}\right],
$$

where $s_{i}^{\prime}=\frac{d}{d t} s_{i}$. Differentiating the equation (2.13) gives

$$
u_{k t}=u^{i i} u_{i i k}+\frac{\partial}{\partial x_{k}} g
$$




$$
u_{k k t}=u^{i i} u_{i i k k}-u^{i i} u^{j j} u_{i j k}^{2}+\log \operatorname{det} \nabla^{2} u+\frac{\partial^{2}}{\partial x_{k}^{2}} g .
$$

It follows

$$
\begin{aligned}
0 \geq & u^{k k} \psi_{k k}-\psi_{t} \\
\geq & \frac{1}{u_{11}}\left(u^{k k} u_{k k 11}-u_{11 t}\right)-\frac{u_{11 k}^{2}}{u_{11}^{2}}+u^{k k}+2 \beta\left\{u_{k k}+\left(u_{i}-s_{i}\right)\left(u^{k k} u_{i k k}-u_{i t}\right)\right. \\
& \left.-n\left(u+s_{n+1}\right)-\left(u+s_{n+1}\right)\left(u_{t}+s_{n+1}^{\prime}\right)+\left(u_{i}-s_{i}\right) s_{i}^{\prime}\right\} \\
\geq & -\frac{1}{u_{11}} \log \operatorname{det} \nabla^{2} u-\frac{1}{u_{11}} \frac{\partial^{2}}{\partial x_{1}^{2}} g+u^{k k}+2 \beta\left\{u_{k k}-\left(u_{i}-s_{i}\right) \frac{\partial}{\partial x_{i}} g\right. \\
& \left.-n\left(u+s_{n+1}\right)-\left(u+s_{n+1}\right)\left(u_{t}+s_{n+1}^{\prime}\right)+\left(u_{i}-s_{i}\right) s_{i}^{\prime}\right\} .
\end{aligned}
$$

Note that

$$
\frac{1}{u_{11}} \frac{\partial^{2}}{\partial x_{1}^{2}} g+2 \beta\left(u_{i}-s_{i}\right) \frac{\partial}{\partial x_{i}} g=g_{p_{1} p_{1}} u_{11}+O(1) .
$$

By (2.16) and (2.17) we therefore conclude

$$
\sum_{k}\left(u_{k k}+u^{k k}\right) \leq C\left(1+\left|u_{t}\right|+\left|s^{\prime}\right|\right) \leq C\left[1+\log \left(u_{k k}+u^{k k}\right)\right],
$$

and so (2.14) follows.

Next we prove (2.15). The upper bound of $H_{t}$ follows from (2.14). We need only to show that $H_{t}$ is bounded from below. Let

$$
q(t)=\int_{S^{n}} x H(x, t) d \sigma .
$$

Then $H(x, t)-q(t) \cdot x>2 \varepsilon$ for some $\varepsilon>0$ depending only on $n, r(X(\cdot, t))$ and $R(X(\cdot, t))$.

Let

$$
\varphi(x, t)=\frac{H_{t}}{H-x \cdot q(t)-\varepsilon} .
$$

Suppose the infimum $\inf \left\{\varphi, x \in S^{n}, t \in[0, T]\right\}$ is attained at $\left(x_{0}, t_{0}\right)$. Without loss of generality we may suppose $x_{0}$ is the south pole and $t_{0}>0$, and $\left(H_{\alpha \beta}\right)$ is diagonal at this point. Then

$$
\psi(y, t)=\frac{u_{t}(y, t)}{u-q(t) \cdot \widetilde{y}-\varepsilon \sqrt{1+|y|^{2}}}, \quad \widetilde{y}=(y,-1),
$$

attains its minimum at $\left(0, t_{0}\right)$, where $u(y, t)=H(y,-1, t)$. We have, at $\left(0, t_{0}\right)$,

$$
\begin{aligned}
& 0 \geq \psi_{t}=\frac{u_{t t}}{u+q_{n+1}(t)-\varepsilon}-\frac{u_{t}\left(u_{t}+q_{n+1}^{\prime}\right)}{\left(u+q_{n+1}(t)-\varepsilon\right)^{2}}, \\
& 0=\psi_{k}=\frac{u_{t k}}{u+q_{n+1}(t)-\varepsilon}-\frac{u_{t}\left(u_{k}-q_{k}(t)\right)}{\left(u+q_{n+1}(t)-\varepsilon\right)^{2}},
\end{aligned}
$$

and

$$
0 \leq \psi_{k k}=\frac{u_{t k k}}{u+q_{n+1}(t)-\varepsilon}-\frac{u_{t} u_{k k}-\varepsilon u_{t}}{\left(u+q_{n+1}(t)-\varepsilon\right)^{2}} .
$$


Differentiating the equation (2.13) yields

$$
u_{t t}=u^{i j} u_{i j t}+\frac{\partial}{\partial t} g=u^{i j} u_{i j k}+g_{u} u_{t}+g_{p_{k}} u_{k t} .
$$

It follows

$$
\begin{aligned}
0 & \leq\left[\sum u^{k k} \psi_{k k}-\psi_{t}\right]\left(u+q_{n+1}(t)-\varepsilon\right)^{2} \\
& \leq \varepsilon u_{t} \sum u^{k k}-n u_{t}+u_{t}\left(u_{t}+q_{n+1}^{\prime}\right)-g_{u} u_{t}\left(u+q_{n+1}-\varepsilon\right)-g_{p_{k}} u_{t}\left(u_{k}-q_{k}\right) .
\end{aligned}
$$

We may suppose $u_{t}$ is negative at $\left(0, t_{0}\right)$, otherwise we are through. From $(2.14)$, we have $H_{t} \leq C$. By the definition of $q(t)$,

$$
|d q / d t| \leq C \sup _{x \in S^{n}}\left|H_{t}\right| \leq C\left(1-\inf H_{t}\right)
$$

We obtain

$$
\begin{aligned}
\sum u^{k k} & \leq C \varepsilon^{-1}\left(1+\left|u_{t}\right|+\left|q_{n+1}^{\prime}\right|\right) \\
& \leq C \varepsilon^{-1}\left(1+\left|u_{t}\right|\right) \leq C \varepsilon^{-1}\left(1+\log \sum u^{k k}\right) .
\end{aligned}
$$

Consequently, $\sum u^{k k} \leq C$, and so $u_{t} \geq-C-\log \Sigma u^{k k} \geq-C$.

The proof of Lemmas 2.1 and 2.2 has followed the line in [4]. From (2.14) and (2.15) we have

$$
H_{\alpha \alpha}(x, t)+H(x, t) \geq C \exp \left(H_{t}\right) \cdot\left[\sup \left(H_{\beta \beta}+H\right)\right]^{1-n} \geq C .
$$

(2.18) gives an upper bound for the principal curvature of $X(\cdot, t)$.

From Lemma 2.2 it is now standard to derive the $C^{3+\alpha, 2+\alpha / 2}$ a priori estimates for $H(x, t)$. On the other hand, the upper bound for $R(X(\cdot, t))$ can be estimated as follows. Let $\widetilde{X}(\cdot, t)$ be a family of spheres with center at $p\left(X_{0}\right)$, the support center of $X_{0}$, so that their radii $\rho(t)$ satisfy

$$
\frac{d}{d t} \rho(t)=n \log \rho(t)+\log \Lambda, \quad \rho(0)=\rho_{0},
$$

where $\Lambda=\sup f(x)$ and $\rho_{0}$ is chosen large enough so that $X_{0} \leq B_{\rho_{0}}\left(p\left(X_{0}\right)\right)$. By the comparison principle it follows

$$
X(\cdot, t) \subset B_{\rho(t)}\left(p\left(X_{0}\right)\right) .
$$

Similarly we have a positive lower bound of $r(X(\cdot, t))$ locally for $t>0$. We thus obtain

Theorem 2.1. For each $X_{0} \in C^{4}$, the equation (2.11) admits a unique solution $X(x, t) \in C^{3+\alpha, 2+\alpha / 2}\left(S^{n} \times[0, T)\right)$ on some maximal time interval $[0, T)$ for some $\alpha \in(0,1)$, and either $r(X(\cdot, t)) \rightarrow 0$ or $R(X(\cdot, t)) \rightarrow+\infty$ as $t \rightarrow T$.

Remark 2.1. By Lemma 2.2 and Krylov's Hölder estimates for uniformly parabolic equations, and by approximation we see that (2.11) admits a unique solution $X(\cdot, t) \in C^{3+\alpha, 2+\alpha / 2}\left(S^{n} \times(0, T)\right)$ provided $X_{0} \in C^{1,1}$.

Remark 2.2. Let $\rho_{0}<\Lambda^{-1 / n}$, then the solution $\rho(t)$ of $(2.19)$ decreases and tends to zero in finite time. Hence if $R\left(X_{0}\right)<\Lambda^{-1 / n}$, we have $R(X(\cdot, t))<R\left(X_{0}\right)$ for $t>0$. In particular, if the solution $X(\cdot, t)$ exists for all $t \in[0, \infty)$, we have

$$
R(X(\cdot, t)) \geq \Lambda^{-1 / n} \quad \forall t>0 .
$$




\section{Proof of Theorem 1}

Lemma 3.1. Let $A=A(X)$ denote the supremum of the radii of the principal curvatures of $X$. We have

$$
r(X) \geq R^{2}(X) / 8 A .
$$

Proof. Let $H$ be the support function of $X$. Without loss of generality we may suppose the infimum $r(X)=\inf _{x \in S^{n}}[H(x)+H(-x)]$ is attained at $x_{0}=(1,0, \cdots, 0)$. Let $\widetilde{R}=\sup _{x \in S^{n}}\left\{H(x)+H(-x),\left\langle x, x_{0}\right\rangle=0\right\}$ and suppose $\widetilde{R}$ is attained at $(0,1,0, \cdots, 0)$. Then $\widetilde{R} \geq \frac{1}{\sqrt{2}} R(X)$. Let $\ell$ be the boundary of the projection of $X$ on the $x_{1} x_{2}$-plane. It is easy to see that the infimum of the curvature of $\ell$ is less than $4 r(X) / \widetilde{R}^{2}$. Namely, the supremum of the radius of curvature of $\ell$ is greater than $\widetilde{R}^{2} / 4 r(X)$. On the other hand, the supremum of the principal radii of $X$ is greater than or equal to that of $\ell$. Hence (3.1) holds.

Lemma 3.2. Let $X$ be a convex hypersurface, and $H$ its support function. We have

$$
I_{n-1}(X)=\int_{S^{n}} H d \sigma \geq \frac{\omega_{n-1}}{n} R(X),
$$

where $\omega_{n-1}$ is the area of the $(n-1)$-dimensional unit sphere.

Proof. We have

$$
I_{n-1}(X)=\int_{X} \sigma_{n-1}=\int_{S^{n}} \frac{\sigma_{n-1}}{\sigma_{n}} d \sigma
$$

Note that $\sigma_{n-1} / \sigma_{n}$ is equal to the sum of all principal radii of $X$ divided by $n$. It follows

$$
I_{n-1}(X)=\frac{1}{n} \int_{S^{n}} \operatorname{tr}\left(H_{\alpha \beta}+H I\right) d \sigma=\int_{S^{n}} H d \sigma .
$$

By proper choice of the coordinates we may suppose $X$ contains the line segment $\ell=\left\{t e_{n+1}, \quad t \in\left(-\frac{1}{2} R(X), \frac{1}{2} R(X)\right)\right\}$. Then $H(x) \geq \frac{1}{2} R(X) \sin \theta$ and

$$
\begin{aligned}
\int_{S^{n}} H(x) d \sigma & \geq \frac{1}{2} R(X) \int_{S^{n}} \sin \theta d \sigma \\
& =R(X) \int_{0}^{\pi / 2} \omega_{n-1} \cos ^{n-1} \theta \sin \theta d \theta=\frac{\omega_{n-1}}{n} R(X),
\end{aligned}
$$

where $\theta$ is the angle between ox $\left(x \in S^{n}\right)$ and the plane $\left\{x_{n+1}=0\right\}$.

From (3.2) it follows

$$
J(X) \geq \frac{\omega_{n-1}}{n} R(X)-\frac{\omega_{n}}{n+1} \Lambda R(X)^{n+1} .
$$

Lemma 3.3. Let $X$ be a solution of (1.1). Then

$$
\Lambda^{-1 / n} \leq R(X) \leq \frac{n}{\omega_{n-1}}\left[J(X)+\frac{\omega_{n} \Lambda}{\lambda^{(n+1) / n}}\right]
$$

where $\Lambda=\sup _{x \in X} f(x), \lambda=\inf _{x \in X} f(x)$. 
Proof. Let $B_{R}\left(x_{0}\right)$ be a ball of smallest radius so that $X \subset \overline{B_{R}\left(x_{0}\right)}$. Then $R^{-n} \leq$ $\Lambda=\sup f(x)$, namely, $R(X) \geq \Lambda^{-1 / n}$. Similarly let $B_{r}\left(y_{0}\right)$ be a ball of largest radius so that $B_{r}\left(y_{0}\right) \subset C l(X)$, we find $r^{-n} \geq \lambda=\inf f(x)$, and so $r(X) \leq \lambda^{-1 / n}$.

Observe that

$$
\int_{X} f(x) d \sigma=\int_{X} K d \sigma=\int_{S^{n}} d \sigma=\omega_{n} .
$$

We have

$$
\omega_{n} \Lambda^{-1} \leq \operatorname{mes}(X) \leq \omega_{n} \lambda^{-1} .
$$

By the Alexandrov-Fenchel inequality [1],

$$
\operatorname{mes}(X) \geq \omega_{n}^{1 /(n+1)}[\operatorname{Vol}(X)]^{n /(n+1)} .
$$

We obtain $\operatorname{Vol}(X) \leq \omega_{n} \lambda^{-(n+1) / n}$. Hence by $(3.2)$,

$$
R(X) \leq \frac{n}{\omega_{n-1}}\left[J(X)+\int_{C l(X)} f(x) d \sigma\right] \leq \frac{n}{\omega_{n-1}}[J(X)+\Lambda \cdot \operatorname{Vol}(X)],
$$

and so (3.4) follows.

Let $X_{0}$ be a smooth and strictly convex hypersurface, and let $X(\cdot, t)$ be the solution of

$$
\frac{\partial}{\partial t} X=-\log (K / f) \cdot \gamma, \quad X(\cdot, 0)=X_{0} .
$$

Recall that $A(X)=\sup _{\alpha, x}\left\{H_{\alpha \alpha}(x)+H(x)\right\}$, where $H$ is the support function of $X$. By Lemma 3.1 and $(2.14)$ we have $r(X(\cdot, t)) \geq R^{2}(X(\cdot, t)) / 8 \widetilde{C}$. Hence if $R(X(\cdot, t))$ is uniformly bounded for $t>0$ and $r(X(\cdot, t)) \rightarrow 0$, then $R(X(\cdot, t)) \rightarrow 0$. It is easy to see that if $R(X(\cdot, t))$ tends to zero, it tends to zero in finite time.

We say $X(\cdot, t)$ shrinks to a point in finite time if $R(X(\cdot, t)) \rightarrow 0$. Otherwise we say $X(\cdot, t)$ exists for all $t>0$.

Lemma 3.4. Suppose the solution $X(\cdot, t)$ exists for all $t>0$. If $R(X(\cdot, t))$ and the support center $p(X(\cdot, t))$ are uniformly bounded, then there exists a subsequence of $X(\cdot, t)$ which converges to a solution of (1.1).

Proof. By assumptions, $X(\cdot, t) \subset B_{R}(0)$ for some $R>0$ large enough. Hence $\log f(x)$ is bounded. Let $H(x, t)$ be the support function of $X(\cdot, t)$. By the a priori estimates in Section 2, we have

$$
\|H(x, t)\|_{C^{3+\alpha, 2+\alpha / 2}\left(S^{n} \times[0, \infty)\right)} \leq M
$$

for some $M>0$. Hence there exists a subsequence $\left(t_{j}\right)$ so that $H\left(\cdot, t_{j}\right)$ converges to a function $H(x)$. We want to show that $H(x)$ satisfies (2.4).

To do so, it suffices to show that $\frac{\partial}{\partial t} H(\cdot, t) \rightarrow 0$ uniformly for $x \in S^{n}$ as $t \rightarrow \infty$. By (1.7) we have

$$
\begin{aligned}
\frac{d}{d t} J(X(\cdot, t)) & =\int_{X}(K-f) H_{t} d \sigma \\
& =-\int_{S^{n}}\left(\frac{f}{K}-1\right) H_{t} d \sigma=-\int_{S^{n}} H_{t}\left(e^{H_{t}}-1\right) d \sigma \leq 0 .
\end{aligned}
$$

By the smoothness of $H(x, t), J(X(\cdot, t))$ is uniformly bounded for $t>0$. Hence $\frac{d}{d t} J(X(\cdot, t)) \rightarrow 0$ as $t \rightarrow \infty$. From (3.6) and by the smoothness of $H_{t}$, we conclude that $H_{t}(x, t) \rightarrow 0$ uniformly for $x \in S^{n}$. This completes the proof. 
Theorem 3.1. If there exists a stable solution of (1.1), then there exists an unstable solution of (1.1).

Proof. Let $\chi$ denote the set of all $C^{1,1}$ convex hypersurfaces with the topology induced by the distance

$$
d\left(X_{1}, X_{2}\right)=\sup _{p_{1} \in X_{1}} \inf _{p_{2} \in X_{2}}\left|p_{1}-p_{2}\right| .
$$

For any $X \in \chi$, we denote by $A(X)$ the supremum of the principal radii of $X$, and by $B(X)$ the supremum of the principal curvatures of $X$. Recall that $A(X)=$ $\sup _{\alpha, x}\left\{H_{\alpha \alpha}(x)+H(x)\right\}$.

Let $X_{0}$ be a stable solution of (1.1). Let $\Phi=\left\{\varphi(s) \in C\left(\left[\delta_{0}, \bar{s}\right)\right], \chi\right) ; \varphi$ satisfies the following (i)-(v)\}, where $\bar{s}=R\left(X_{0}\right)$.

(i) $\varphi(\bar{s})=X_{0}$;

(ii) $R\left(\varphi\left(\delta_{0}\right)\right)=\delta_{0}, R(\varphi(s))=s$;

(iii) $\varphi\left(s_{1}\right)<\varphi\left(s_{2}\right) \forall s_{1}<s_{2}$;

(iv) $A(\varphi(s)) \leq A_{0}$;

(v) $B(\varphi(s)) \leq B_{0}$;

where $\delta_{0}, A_{0}, B_{0}$ are positive constants to be determined. Set

$$
c_{0}=\inf _{\varphi \in \Phi} \sup _{s \in\left[\delta_{0}, \bar{s}\right]} J(\varphi(s)) .
$$

By (3.3),

$$
c_{0} \geq \sup _{\delta_{0}<R<\bar{s}}\left[\frac{\omega_{n-1}}{n} R-\frac{\omega_{n} \Lambda}{n+1} R^{n+1}\right] .
$$

Take $\delta_{0}$ small enough so that $J\left(\varphi\left(\delta_{0}\right)\right)<c_{0}$ for any $\varphi \in \Phi$. Obviously $c_{0}>0$.

We claim that $c_{0}>J\left(X_{0}\right)$. Indeed, since $X_{0}$ is a stable solution, there exists an open subset $\chi_{0}$ of $\chi$ which contains $X_{0}$ so that $J\left(X_{0}\right)=\inf \left\{J(X) ; X \in \chi_{0}\right\}$, and

$$
J\left(X_{0}\right) \leq-\varepsilon_{0}+\inf \left\{J(X) ; X \in \partial \chi_{0}, A(X) \leq A_{0}, B\left(X_{0}\right) \leq B_{0}\right\}
$$

for some $\varepsilon_{0}>0$ depending on $A_{0}$ and $B_{0}$. If $J\left(X_{0}\right) \leq \sup \left\{J(X) ; R(X)=\delta_{0}\right\}$, then we have already $J\left(X_{0}\right)<c_{0}$ since $\delta_{0}$ is small enough. Otherwise there exists an $s_{0} \in\left(\delta_{0}, \bar{s}\right)$ so that $\varphi\left(s_{0}\right) \in \partial \chi_{0}$. Hence $\sup _{s \in\left(\delta_{0}, \bar{s}\right)} J(\varphi(s)) \geq \varepsilon_{0}+J\left(X_{0}\right)$ for any $\varphi \in \Phi$, and so $c_{0} \geq \varepsilon_{0}+J\left(X_{0}\right)$.

For $\varepsilon>0$ small enough, let $\varphi(s) \in \Phi$ so that $\sup _{s \in\left[\delta_{0}, \bar{s}\right]} J(\varphi(s)) \leq c_{0}+\varepsilon$. We consider the logarithmic gradient flow:

$$
\frac{\partial}{\partial t} X=-\log (K / f) \cdot \gamma, \quad X(0)=\varphi(s)
$$

For any given $s \in\left[\delta_{0}, \bar{s}\right],(3.9)$ admits a unique solution $X_{s}(\cdot, t)$ on a maximal time interval $\left[0, T_{s}\right)$; and $X_{s}\left(\cdot,\left.t\right|_{s=\bar{s}} \equiv X_{0}\right.$ since $X_{0}$ is a solution of (1.1). By the a priori estimates in Section $2, X_{s}(\cdot, t)$ depends continuously on $s$. In particular, if $X_{s_{0}}(\cdot, t)$ shrinks to a point in finite time, then $X_{s}(\cdot, t)$ also shrinks to a point in finite time for $s$ near $s_{0}$.

By the comparison principle we have $X_{s_{1}}(\cdot, t)<X_{s_{2}}(\cdot, t)$ for any $s_{1}<s_{2}$. By Remark 2.2, we have $R\left(X_{\delta_{0}}(\cdot, t)\right) \leq \delta_{0}$ for $t>0$ since $\delta_{0}<\Lambda^{-1 / n}$. For any $t>0$, let $\varphi_{t}(s)=X_{s^{\prime}}(\cdot, t)$, where $s^{\prime}$ is uniquely determined by $R\left(X_{s^{\prime}}(\cdot, t)\right)=s$. Then $\varphi_{t}(s)$ satisfies (i)-(iii) above. We may choose $A_{0}$ and $B_{0}$ large enough, so that $\varphi_{t}(s)$ satisfies (iv) and (v). Indeed, for any given $t_{0}>0$, let $A^{*}=\sup \left\{A\left(\varphi_{t}(s)\right) ; \quad t \in\right.$ $\left.\left[0, t_{0}\right], s \in\left[\delta_{0}, \bar{s}\right]\right\}$. If $A^{*}$ is attained at $t=0$, then (iv) holds. If $A^{*}$ is attained 
at $t>0$, by (2.14) we have $A^{*} \leq \widetilde{C}=\widetilde{C}\left(n, f, \delta_{0}, \bar{s}\right)$. Hence (iv) holds if $A_{0}$ is sufficiently large. By virtue of Lemma 3.1, we have $r\left(X_{s}(\cdot, t)\right) \geq r_{0}$ for some $r_{0}>0$ depending only on $A_{0}, \delta_{0}$ and $\bar{s}$. Hence by $(2.15)$ and (2.18) we may also choose $B_{0}$ sufficiently large so that $(\mathrm{v})$ holds for any $t>0$.

By (3.6) it therefore follows

$$
c_{0} \leq \sup _{s \in\left[\delta_{0}, \bar{s}\right]} J\left(\varphi_{t}(s)\right) \leq \sup _{s \in\left[\delta_{0}, \bar{s}\right]} J(\varphi(s)) \leq c_{0}+\varepsilon
$$

Take $s(t)$ so that $X_{s(t)}(\cdot, t)=\varphi_{t}\left(\delta_{0}\right)$, namely, $R\left(X_{s(t)}(\cdot, t)\right)=\delta_{0}$. We claim that there exists $\varepsilon_{1}>0$ so that $s(t) \leq \bar{s}-\varepsilon_{1}$ for all $t>0$. Indeed, let $\varepsilon_{1}>0$ small so that

$$
J(\varphi(s))<\frac{1}{2} \varepsilon_{0}+J\left(X_{0}\right) \quad \forall s \in\left[\bar{s}-\varepsilon_{1}, \bar{s}\right],
$$

where $\varepsilon_{0}$ is the constant in (3.8). If $X_{\bar{s}-\varepsilon_{1}}(\cdot, t)$ shrinks to a point in finite time, then there exists a $t_{0} \geq 0$ so that $X_{\bar{s}-\varepsilon_{1}}\left(t_{0}\right) \in \partial \chi_{0}$, which imply $J\left(X_{\bar{s}-\varepsilon_{1}}\left(t_{0}\right)\right) \geq$ $\varepsilon_{0}+J\left(X_{0}\right)$. On the other hand, by $(3.6)$ we have $J\left(X_{\bar{s}-\varepsilon_{1}}(\cdot, t)\right) \leq J\left(X_{\bar{s}-\varepsilon_{1}}(\cdot, 0)\right) \leq$ $\frac{1}{2} \varepsilon_{0}+J\left(X_{0}\right) \forall t>0$, a contradiction.

By (3.11), we have

$$
J\left(X_{s}(\cdot, t)\right) \leq \frac{1}{2} \varepsilon_{0}+J\left(X_{0}\right) \quad \forall s \in\left[\bar{s}-\varepsilon_{1}, \bar{s}\right] \text { and } t>0 .
$$

Suppose the supremum $\sup \left\{J\left(X_{s}(\cdot, t)\right) ; s \in\left(\delta_{0}, \bar{s}\right)\right\}$ is attained at $s^{*}(t)$. By (3.12), $s^{*}(t) \leq \bar{s}-\varepsilon_{1}$. Extract a subsequence of $s^{*}(t)$ so that it converges to $s^{*}$. Then $s^{*} \leq \bar{s}-\varepsilon_{1}$. For any $t>0$, let $I(t)=\left\{s \in\left[\delta_{0}, \bar{s}\right] ; \quad J\left(X_{s}(\cdot, t)\right) \geq c_{0}\right\} . I(t)$ is a nonempty closed subset of $\left[\delta_{0}, \bar{s}\right]$. By $(3.6)$ we have $I\left(t_{1}\right) \supset I\left(t_{2}\right)$ for any $t_{1} \leq t_{2}$. Let $I=\bigcap\{I(t), t>0\}$. Then for any $s \in I$, we have $J\left(X_{s}(\cdot, t)\right) \geq c_{0} \quad \forall t>0$. In particular, $J\left(X_{s^{*}}(\cdot, t)\right) \geq c_{0}$ for any $t>0$. We also claim that $X_{s^{*}}(\cdot, t)$ can not shrink to a point in finite time. For if it did so, there would exist a neighbourhood $N$ of $s^{*}$ so that for every $s \in N, X_{s}(\cdot, t)$ would shrink to a point in finite time. This is impossible by the definition of $s^{*}$.

Note that $X_{s^{*}}(\cdot, t) \leq X_{0}$. By Lemma 3.4, there is a subsequence $t_{j} \rightarrow \infty$ so that $X_{s^{*}}\left(\cdot, t_{j}\right)$ converges to a solution $X_{\varepsilon}$ of (1.1). By (3.10) we have $c_{0} \leq J\left(X_{\varepsilon}\right) \leq c_{0}+\varepsilon$. Let $\varepsilon \rightarrow 0$, and by (2.10) and Lemma 3.3, we may extract a subsequence of $X_{\varepsilon}$ so that it converges to a solution $X$ of (1.1) with $J(X)=c_{0}$. This completes the proof.

Remark 3.1. In the above proof, we require that $\varphi(s)$ satisfies (iv) and (v) is in order that (3.8) holds. Note that in the definition for the stability of solutions we only require that $J(\widetilde{X})>J\left(X_{0}\right)$ for any $\widetilde{X} \in \partial \chi_{0}$, which does not imply $\inf \left\{J(\widetilde{X}), \widetilde{X} \in \partial \chi_{0}\right\}>J\left(X_{0}\right)$ immediately.

Corollary 3.1. Suppose $f(x)$ is integrable over $\mathbb{R}^{n+1}$ and there exists a convex hypersurface $X_{0}$ so that $J\left(X_{0}\right) \leq 0$. Then equation (1.1) admits at least two solutions.

Proof. In [10] Tso proved that under the above conditions, equation (1.1) has a solution $X_{0}$ which reaches the absolute minimum of $J$. By Theorem 3.1, there exists an unstable solution $X^{*}$ of (1.1) so that $X^{*}<X_{0}$.

Corollary 3.2. Suppose $f(x)|x|^{n} \rightarrow 0$ uniformly as $|x| \rightarrow \infty$. Then there exists $\theta_{*}>0$ so that the problem

$$
K(p)=\theta f(p), \quad p \in X
$$


has no solution for $\theta \in\left(0, \theta_{*}\right)$, has at least one solution for $\theta=\theta_{*}$, and admits at least two solutions for $\theta>\theta_{*}$.

Proof. Step 1. We prove that for any given $\bar{\theta}>0$, there exists $\bar{R}>0$ so that if $X$ is a solution of $(1.1)_{\theta}$ with $\theta \in(0, \bar{\theta})$, then $X \subset B_{\bar{R}}(0)$.

Indeed, let $H$ be the support function of the solution $X$. Suppose the supremum $M=\sup \left\{H(x), x \in S^{n}\right\}$ is attained at $e_{1}=(1,0, \cdots, 0)$. Let $Z=\left\{x \in X ; x_{1}>\right.$ $\left.\frac{1}{2} M\right\}$. It is easy to see that $G(Z) \supset\left\{x \in S^{n} ; x_{1}>\frac{3}{4}\right\}$, where $G: X \rightarrow S^{n}$ is the Gauss map of $X$. Hence

$$
\operatorname{mes}(G(Z)) \geq C>0
$$

for some $C$ depending only on $n$. On the other hand,

$$
\operatorname{mes}(G(Z))=\int_{G(Z)} d \sigma=\int_{Z} K d \sigma=\int_{Z} \theta f d \sigma .
$$

Note that $\operatorname{mes}(Z) \leq \operatorname{mes}(X) \leq \omega_{n} R(X)^{n} \leq 2^{n} \omega_{n} M^{n}$, and that $f(x)=o\left(|x|^{-n}\right)=$ $o\left(M^{-n}\right)$ on $Z$ for $M$ large. We have

$$
\operatorname{mes}(G(Z))=\theta \int_{Z} f(x) d \sigma \leq \bar{\theta} \int_{Z} f(x) \rightarrow 0
$$

as $M \rightarrow \infty$. Hence there exists $\bar{R}>0$ so that $M \leq \bar{R}$, namely, $X \subset B_{\bar{R}}(0)$.

Consequently $(1.1)_{\theta}$ has no solution for $\theta>0$ small. For if $X$ is a solution of $(1.1)_{\theta}$, we have $R(X) \geq(\theta \Lambda)^{-1 / n}$, where $\Lambda=\sup f(x)$.

Step 2. Let

$$
\theta_{*}=\inf \left\{\theta ; \quad(1.1)_{\theta} \text { has at least one solution }\right\} .
$$

By step 1, we have $\theta_{*}>0$. On the other hand, by Lemma 3.2 we have

$$
J_{\theta}(X)=I_{n-1}(X)-\theta \int_{C l(X)} f(x) d x \geq \frac{\omega_{n-1}}{n} R(X)-o(R(X))
$$

as $R(X)$ large enough. Hence $J_{\theta}(X)$ is bounded from below. Obviously inf $J_{\theta}(X) \leq$ 0 for $\theta$ large. Hence by the argument of Tso [10], (1.1) $)_{\theta}$ has a solution which reaches the absolute minimum of $J_{\theta}(X)$. Thus $\theta_{*}<+\infty$.

Let $\theta_{j} \rightarrow \theta_{*}$ and $X_{\theta_{j}}$ be a solution of $(1.1)_{\theta}$ with $\theta=\theta_{j}$. From step 1 we have $X_{\theta_{j}} \subset B_{\bar{R}}(0)$ for some $\bar{R}>0$. On the other hand, we have $R\left(X_{\theta_{j}}\right) \geq\left(\theta_{j} \Lambda\right)^{-1 / n}$. Hence by $(2.10)$, there exists a subsequence of $X_{\theta_{j}}$ which converges to a solution $X_{*}$ of $(1.1)_{\theta}$ with $\theta=\theta_{*}$.

Step 3. Let $\chi_{0}=\left\{X \in \chi ; X>X_{*}\right\}$. For any $\theta>\theta_{*}$, we want to show that $(1.1)_{\theta}$ has a stable solution in $\chi_{0}$, which implies by Theorem 3.1 that $(1.1)_{\theta}$ has at least two solutions for $\theta>\theta_{*}$.

Let $c_{\theta}=\inf \left\{J_{\theta}(X) ; \quad X \in \chi_{0}\right\}$. For any $\varepsilon>0$, choose $X_{0} \in \chi_{0}$ so that $J_{\theta}\left(X_{0}\right)<c_{\theta}+\varepsilon$. Let $X(\cdot, t)$ be the solution of (3.5). By the comparison principle, $X(\cdot, t)>X_{*}$. From $(3.6)$ we have $J_{\theta}(X(\cdot, t)) \leq c_{\theta}+\varepsilon$. By $(3.2)$,

$$
R(X(\cdot, t)) \leq \frac{n}{\omega_{n-1}}\left[J_{\theta}(X(\cdot, t))+\theta\|f\|_{L^{1}\left(B_{R(X)}(0)\right)}\right] \leq \frac{n}{\omega_{n-1}}\left[c_{\theta}+1+o(R(X(\cdot, t)))\right] .
$$

Namely, $R(X(\cdot, t)) \leq C$. Hence by Lemma 3.4 , there exists a subsequence $X\left(\cdot, t_{j}\right)$ which converges to a solution $X_{\varepsilon}$ of $(1.1)_{\theta}$ with $J_{\theta}\left(X_{\varepsilon}\right)<c_{\theta}+\varepsilon$. Let $\varepsilon \rightarrow 0$, by (2.10) and Lemma 3.3, we obtain a solution $X_{\theta}$ which satisfies $J\left(X_{\theta}\right)=c_{\theta}$. 
It remains to show that $c_{\theta}<J\left(X_{0}\right)$ for any $X_{0} \in \partial \chi_{0}$. Note that $X_{0} \in \partial \chi_{0}$ if and only if $X_{*} \leq X_{0}$ and $X_{*} \cap X_{0}$ is not empty. It is easy to see that for any $X_{0} \in \partial \chi_{0}, X_{0}$ is not a solution of $(1.1)_{\theta}$ since $\theta>\theta_{*}$. Let $X(\cdot, t)$ be the solution of (3.5) with $X_{0} \in \partial \chi_{0}$. By the comparison principle we have $X(\cdot, t)>X_{*}$ and by (3.6), $\frac{d}{d t} J(X(\cdot, t))<0$. Hence $J\left(X_{0}\right)>J\left(X_{\theta}\right)$.

Corollary 3.3. Suppose $f(x)$ satisfies (1.2). Then (1.1) admits at least two solutions.

Proof. It suffices to show that (1.1) has a stable solution.

Let $\chi_{0}=\left\{X \in \chi ; \quad B_{R_{1}}(0) \subset \subset C l(X) \subset B_{R_{2}}(0)\right\} . \chi_{0}$ is an open subset of $\chi$, and $X \in \chi$ is a boundary element of $\chi_{0}$ if and only if $B_{R_{1}}(0) \subset C l(X) \subset \bar{B}_{R_{2}}(0)$ and there exists a point $p \in X$ so that $p \in \partial B_{R_{2}}(0)$ or $p \in \partial B_{R_{1}}(0)$.

For any $\varepsilon>0$, take $X_{0} \in \chi_{0}$ so that $J\left(X_{0}\right) \leq \inf \left\{J(X) ; \quad X \in \chi_{0}\right\}+\varepsilon$. Let $X(\cdot, t)$ be the solution of (3.5). By the condition (1.2), we have $B_{R_{1}}(0) \subset$ $C l(X(\cdot, t)) \subset B_{R_{2}}(0)$. Hence by Lemma 3.4, there exists a subsequence $t_{j}$ so that $X\left(\cdot, t_{j}\right)$ converges to a solution $X_{\varepsilon}$ of (1.1). Let $\varepsilon \rightarrow 0$, we obtain a subsequence of $X_{\varepsilon}$ which converges to a solution $X_{*} \in \chi_{0}$ of (1.1) with $J\left(X_{*}\right)=\inf \{J(X) ; X \in$ $\left.\chi_{0}\right\}$. Similar to the proof of Corollary 3.2 we have $J\left(X_{*}\right)<J(X)$ for any $X \in \partial \chi_{0}$. Hence $X_{*}$ is a stable solution.

Next we prove that if $f(x)$ satisfies (1.2) and (1.5), (1.1) has at least three solutions.

Lemma 3.5. Let $X(\cdot, t)$ be a solution of (3.5). We have

$$
\frac{d}{d t} \operatorname{Vol}(X(\cdot, t)) \geq \omega_{n}^{1 /(n+1)}[\operatorname{Vol}(X(\cdot, t))]^{n /(n+1)}-\frac{4}{\lambda} \omega_{n}
$$

Proof. Let

$$
h(t)=\frac{1}{n+1} \int_{X(\cdot, t)} H d \sigma=\frac{1}{n+1} \int_{S^{n}} H \operatorname{det}\left(H_{\alpha \beta}+H I\right) d \sigma
$$

be the volume enclosed by $X(\cdot, t)$, where $H(\cdot, t)$ is the support function of $X(\cdot, t)$. We have

$$
h^{\prime}(t)=\int_{S^{n}} H_{t} \operatorname{det}\left(H_{\alpha \beta}+H I\right) d \sigma=\int_{X(\cdot, t)} H_{t} d \sigma .
$$

On $X^{-}=X \cap\left\{H_{t}<0\right\}$, we have

$$
\int_{X^{-}} H_{t} d \sigma=-\int_{X^{-}} \log \frac{K}{f} d \sigma \geq-\int_{X^{-}} \frac{K}{f} d \sigma \geq-\int_{S^{n}} \frac{1}{f} d \sigma \geq-\frac{\omega_{n}}{\lambda} .
$$

Hence

$$
h^{\prime}(t) \geq-\omega_{n} / \lambda .
$$

For $a>0$ let $X_{a}=X \cap\{K \leq a\}$. Note that $\int_{X} K d \sigma=\int_{S^{n}} d \sigma=\omega_{n}$. We have $\operatorname{mes}\left(X \backslash X_{a}\right) \leq \omega_{n} / a$, i.e., $\operatorname{mes}\left(X_{a}\right) \geq \operatorname{mes}(X)-\omega_{n} / a$. Let $a=\lambda / e$, then $H_{t}=-\log (K / f) \geq 1$ on $X_{a}$. Note that $X_{a} \cap X^{-}=\emptyset$, we have

$$
h^{\prime}(t) \geq \int_{X_{a}} H_{t} d \sigma+\int_{X^{-}} H_{t} d \sigma \geq \operatorname{mes}(X)-\frac{e \omega_{n}}{\lambda}-\frac{\omega_{n}}{\lambda} .
$$

By the Alexandrov-Fenchel inequality [1],

$$
\operatorname{mes}(X) \geq \omega_{n}^{1 /(n+1)}[\operatorname{Vol}(X)]^{n /(n+1)},
$$


we obtain

$$
h^{\prime}(t) \geq \omega_{n}^{1 /(n+1)}\left[\operatorname{Vol}(X(\cdot, t)]^{n /(n+1)}-\frac{4}{\lambda} \omega_{n} .\right.
$$

Lemma 3.5 follows.

Lemma 3.6. Let $X_{0}=\left\{\sum_{i=1}^{n} \frac{x_{i}^{2}}{a^{2}}+\frac{x_{n+1}^{2}}{M^{2}}\right\}$ be an ellipsoid, where $a>0$ is a fixed constant. Let $X(\cdot, t)$ be the solution of

$$
\frac{\partial}{\partial t} X=-\log (K / \lambda) \cdot \gamma, \quad X(\cdot, 0)=X_{0} .
$$

Then there exists $M_{0}>0$ so that if $M>M_{0}$, we have $r(X(\cdot, t)) \rightarrow \infty$ as $t \rightarrow+\infty$.

Proof. For simplicity we suppose $\lambda=1$. It is easy to see that $X(\cdot, t)$ is symmetric with respect to the $x_{n+1}$-axis and the plane $\left\{x_{n+1}=0\right\}$. Let $b<\frac{1}{2} M$ be a constant to be determined. Let $F_{t}=\left\{x \in X(\cdot, t) ; \quad\left|x_{n+1}\right|<b\right\}, G_{t}=\{x \in$ $\left.C l(X(\cdot, t)) ; \quad\left|x_{n+1}\right|<b\right\}$. Then

$$
\operatorname{Vol}\left(C l(X(\cdot, t)) \backslash G_{t}\right) \geq C_{n} a^{n} M .
$$

Similar to (3.14), we have $\frac{d}{d t} \operatorname{Vol}\left(C l(X(\cdot, t)) \backslash G_{t}\right) \geq-\omega_{n}$. Hence $\operatorname{Vol}\left(C l(X(\cdot, t)) \backslash G_{t}\right)$ $>0$ when $t \leq C_{n} a^{n} M / \omega_{n}$.

If the unit sphere $S^{n}$ is strictly contained in $X\left(\cdot, t_{0}\right)$, then by the comparison principle we have $B_{\rho(t)}(0) \subset C l(X(\cdot, t))$, where $\rho(t)$ satisfies

$$
\rho^{\prime}(t)=n \log \rho(t), \rho\left(t_{0}\right)=r\left(X\left(\cdot, t_{0}\right)\right) .
$$

Hence $r(X(\cdot, t)) \rightarrow \infty$.

We claim that if $M$ is large enough, $X(\cdot, t)$ contains the unit sphere $S^{n}$ provided $t$ is suitably large, from which it follows $r(X(\cdot, t)) \rightarrow \infty$. Indeed, similar to (3.15) we have

$$
\frac{d}{d t} \operatorname{Vol}\left(G_{t}\right) \geq \operatorname{mes}\left(F_{t}\right)-4 \omega_{n}
$$

By the Alexandrov-Fenchel inequality we have

$$
\operatorname{mes}\left(F_{t}\right)+2 \omega_{n-1} \geq \operatorname{mes}\left(\partial G_{t}\right) \geq \omega_{n}^{1 /(n+1)}\left[\operatorname{Vol}\left(G_{t}\right)\right]^{n /(n+1)} .
$$

Hence

$$
\frac{d}{d t} \operatorname{Vol}\left(G_{t}\right) \geq \omega_{n}^{1 /(n+1)}\left[\operatorname{Vol}\left(G_{t}\right)\right]^{n /(n+1)}-4 \omega_{n}-2 \omega_{n-1} .
$$

Note that if $M$ is large enough, we have $\operatorname{Vol}\left(G_{0}\right)>\frac{\omega_{n-1}}{2 n} a^{n} b$ by the convexity of $G_{0}$. Take $b>1$ large enough so that $\frac{\omega_{n-1}}{2 n} a^{n} b>\left(1+4 \omega_{n}+2 \omega_{n-1}\right)^{(n+1) / n} \omega_{n}^{-1 / n}$. Then $\frac{d}{d t} \operatorname{Vol}\left(G_{t}\right) \geq 1$ and we deduce that

$$
\operatorname{Vol}\left(G_{t}\right) \geq \operatorname{Vol}\left(G_{0}\right)+t>2 b^{n+1} \text { for } t \in\left(2 b^{n+1}, C_{n} a^{n} M / \omega_{n}\right)
$$

which implies $S^{n} \subset G_{t}$ when $b$ is large enough.

Lemma 3.6 implies, by the comparison principle, that if $X(\cdot, t)$ is a solution of (3.5) and if $r\left(X_{0}\right) \geq a, R\left(X_{0}\right) \geq M_{0}$ for some $M_{0}$ large, then $r(X(\cdot, t)) \rightarrow+\infty$.

Lemma 3.7. There exists $M_{1}>0$ depending only on $\lambda, n, M_{0}$ and $C$ so that if $J\left(X_{0}\right)<C$ and $\operatorname{Vol}\left(X_{0}\right)>M_{1}$, the solution $X(\cdot, t)$ of (3.5) satisfies $R(X(\cdot, t))>$ $M_{0}$ for all $t>0$, where $M_{0}$ is the constant in Lemma 3.6. 
Proof. Let $M_{1}=\max \left\{\frac{\omega_{n}}{n+1} M_{0}^{n+1}, \omega_{n}(4 / \lambda)^{(n+1) / n}\right\}$. If $\operatorname{Vol}\left(X_{0}\right)>M_{1}$, by Lemma 3.5 we have $\frac{d}{d t} \operatorname{Vol}(X(\cdot, t)) \geq 0$ and $\operatorname{so} \operatorname{Vol}(X(\cdot, t))>M_{1}$ for all $t>0$. Hence $R(X(\cdot, t)) \geq\left[\frac{n+1}{\omega_{n}} \operatorname{Vol}(X(\cdot, t)]^{1 /(n+1)}>M_{0}\right.$ for all $t>0$.

Theorem 3.2. Suppose $f(x)$ satisfies (1.5). If there exists a stable solution $X_{0}$ of (1.1), then (1.1) has an unstable solution $X_{1}$ so that $X_{0} \subset C l\left(X_{1}\right)$.

Proof. Let $\Phi=\left\{\varphi(s) \in C\left(\left[0, s_{\varphi}\right], \chi\right) ; \varphi\right.$ satisfies the following (i)-(v) $\}$.

(i) $\varphi(0)=X_{0}$;

(ii) $\varphi\left(s_{1}\right)<\varphi\left(s_{2}\right) \quad \forall s_{1}<s_{2}$;

(iii) $A(\varphi(s)) \leq A_{0}$;

(iv) $B(\varphi(s)) \leq B_{0}$;

(v) $R\left(\varphi\left(s_{\varphi}\right)\right) \leq M_{0}$ and $R\left(X_{s_{\varphi}}(\cdot, t)\right) \rightarrow+\infty$ as $t \rightarrow+\infty$;

where $M_{0}$ is the constant in Lemma 3.6, $A_{0}$ and $B_{0}$ are positive constants to be determined, $A(X)$ and $B(X)$ are as in the proof of Theorem 3.1, and $X_{s}(\cdot, t)$ is the solution of

$$
\frac{d}{d t} X=-\log (K / f) \cdot \gamma, \quad X(\cdot, 0)=\varphi_{s}
$$

Note that $\left.X_{s}(\cdot, t)\right|_{s=0}=X_{0}$ for any $t>0$ and $X_{0}<X_{s}(\cdot, t)$ for any $s \in\left(0, s_{\varphi}\right)$ and $t>0$.

We claim that there exist $A_{0}$ and $B_{0}$ large enough so that the solution $X_{s}(\cdot, t)$ of (3.16) satisfies $A\left(X_{s}(\cdot, t)\right) \leq A_{0}$ and $B\left(X_{s}(\cdot, t)\right) \leq B_{0}$ provided $R\left(X_{s}(\cdot, t)\right) \leq$ $M_{0}$. Indeed, if $R\left(X_{s}(\cdot, t)\right) \leq M_{0}$, by Lemma 3.7, we have $\operatorname{Vol}\left(X_{s}\left(\cdot, t^{\prime}\right)\right) \leq M_{1}$ for $t^{\prime} \in(0, t)$. Since $X_{s}(\cdot, t)$ contains $X_{0}$, by (ii) and the comparison principle we have $R\left(X_{s^{\prime}}\left(\cdot, t^{\prime}\right)\right) \leq M_{2}$ for all $s^{\prime} \in(0, s)$ and $t^{\prime} \in(0, t)$. Let $A^{*}=\sup \left\{A\left(X_{s^{\prime}}\left(\cdot, t^{\prime}\right)\right) ; s^{\prime} \in\right.$ $\left.(0, s), t^{\prime} \in(0, t)\right\}$. If the supremum is attained at $t=0$, then $A^{*} \leq A\left(X_{0}\right)$. If it is attained at $t>0$, by (2.14) we have $A^{*} \leq \widetilde{C}=\widetilde{C}\left(n, f, M_{2}\right)$. Hence if $A_{0}$ is large enough, we have $A^{*} \leq A_{0}$. By Lemma 3.1 and $(2.18)$ we can also choose $B_{0}$ large enough so that $B\left(X_{s}(\cdot, t)\right) \leq B_{0}$ provided $R\left(X_{s}(\cdot, t)\right) \leq M_{0}$.

Set

$$
c_{0}=\inf _{\varphi \in \Phi} \sup _{s \in\left[0, s_{\varphi}\right]} J(\varphi(s)) .
$$

We claim $c_{0}>J\left(X_{0}\right)$. Indeed, for any $\varphi \in \Phi$, let

$$
\widetilde{\varphi}(s)= \begin{cases}\varphi(s), & s \in\left(0, s_{\varphi}\right] ; \\ X_{s_{\varphi}}\left(\cdot, s-s_{\varphi}\right), & s \in\left(s_{\varphi},+\infty\right) .\end{cases}
$$

By Lemma 3.6 and (v) we have $r\left(X_{s_{\varphi}}(\cdot, t)\right) \rightarrow+\infty$, and hence $J(\widetilde{\varphi}(s)) \rightarrow-\infty$ as $s \rightarrow+\infty$. From (3.6) we have

$$
\sup \left\{J(\varphi(s)) ; s \in\left(0, s_{\varphi}\right)\right\}=\sup \{J(\widetilde{\varphi}(s)) ; s \in(0,+\infty)\} .
$$

Since $X_{0}$ is a stable solution, there exists an open $\chi_{0} \subset \chi$ which contains $X_{0}$ so that $J(X)>J\left(X_{0}\right) \forall X \in \partial \chi_{0}$. Hence

$$
\inf \left\{J(X) ; \quad X \in \partial \chi_{0}, A(X) \leq A_{0}, B(X) \leq B_{0}\right\} \geq J\left(X_{0}\right)+\varepsilon_{0}
$$

for some $\varepsilon_{0}>0$ depending on $A_{0}$ and $B_{0}$. Note that $\widetilde{\varphi}(s) \cap \partial \chi_{0}$ is not empty. From the above two formulae we therefore conclude that $c_{0}>J\left(X_{0}\right)$. 
For $\varepsilon>0$ small enough, let $\varphi(s) \in \Phi$ so that $\sup _{s \in\left[0, s_{\varphi}\right]} J(\varphi(s)) \leq c_{0}+\varepsilon$, and let $X_{s}(\cdot, t)$ be the solution of $(3.16)$. For any $t>0$, let

$$
s\left(\varphi_{t}\right)=\sup \left\{s \in\left(0, s_{\varphi}\right) ; \quad R\left(X_{s}(\cdot, t)\right) \leq M_{0}\right\},
$$

and let $\varphi_{t}(s)=X_{s}(\cdot, t)$ for $s \in\left(0, s\left(\varphi_{t}\right)\right)$. Then $\varphi_{t}$ is a path in $\Phi$, and

$$
\sup _{s \in\left[0, s\left(\varphi_{t}\right)\right]} J\left(\varphi_{t}(s)\right) \geq c_{0} .
$$

Suppose the supremum $\sup _{s \in\left(0, s\left(\varphi_{t}\right)\right)} J\left(\varphi_{t}(s)\right)$ is attained at $s=s(t)$. Choose a subsequence $t_{j}$ so that $s_{j}=s\left(t_{j}\right) \rightarrow s^{*}$ for some $s^{*} \in\left[0, s_{\varphi}\right)$. We claim $s^{*}>0$. For if not, we would have, by (3.6),

$$
c_{0} \leq J\left(\varphi_{t_{j}}\left(s_{j}\right)\right) \leq J\left(\varphi_{0}\left(s_{j}\right)\right) \rightarrow J\left(\varphi_{0}(0)\right)<c_{0},
$$

a contradiction. We also claim that $X_{s^{*}}(\cdot, t)$ is uniformly bounded for $t>0$. Indeed, if it is not true, by Lemma 3.6 and note that $X_{0}<X_{s^{*}}(\cdot, t)$, we have $r\left(X_{s^{*}}(\cdot, t)\right) \rightarrow \infty$. Hence there exists an $\varepsilon_{0}>0$ so that $r\left(X_{s}(\cdot, t)\right) \rightarrow \infty$ for $s \in N=\left[s^{*}-\varepsilon_{0}, s^{*}+\varepsilon_{0}\right]$, which implies $J\left(X_{s}(\cdot, t)\right) \rightarrow-\infty$ uniformly for $s \in N$. On the other hand, by the choice of $s_{j}, J\left(X_{s_{j}}\left(\cdot, t_{j}\right)\right) \geq c_{0}$. When $j$ is sufficiently large, we reach a contradiction.

Consequently by Lemma 3.4 , there exists a subsequence $t_{j} \rightarrow \infty$ so that $X_{s^{*}}\left(\cdot, t_{j}\right)$ converges to a solution $X^{*}$ of (1.1) with $c_{0} \leq J\left(X^{*}\right) \leq c_{0}+\varepsilon$. Let $\varepsilon \rightarrow 0$ and by (2.10) and Lemma 3.3, we obtain a solution $X$ of (1.1) with $J(X)=c_{0}$.

Corollary 3.4. Suppose $f(x)$ satisfies (1.2) and (1.5). Then (1.1) admits at least three solutions.

Proof. We have shown in Corollary 3.3 that equation (1.1) has a stable solution $X_{0} \in \chi_{0}$. By Theorems 3.1 and 3.2, there exist two unstable solutions $X_{1}$ and $X_{2}$ so that $X_{1}<X_{0}$ and $X_{0}<X_{2}$, respectively.

Theorem 3.1 can be slightly improved. Let $X$ be a solution of (1.1). We say $X$ is relatively stable if there exists an open subset $\chi_{0}$ which contains $X$ so that $J(X)=\inf \left\{J(\widetilde{X}), \tilde{X} \in \chi_{0}\right\}$.

Theorem 3.3. If (1.1) has a relatively stable solution, then it has at least two solutions.

Proof. Let $X_{0}$ be a relatively stable solution of (1.1). Let $\Phi$ and $c_{0}$ be as in the proof of Theorem 3.1. If $c_{0}>J\left(X_{0}\right)$, the proof of Theorem 3.1 is valid and so (1.1) has at least two solutions. If $c_{0}=J\left(X_{0}\right)$, we proceed as follows.

Let $\chi_{0}=\left\{X \in \chi ; \quad \operatorname{dist}\left(X, X_{0}\right)<\rho_{0}\right\}$. Since $X_{0}$ is a relatively stable solution, we may choose $\rho_{0} \in\left(0, \frac{1}{2} \Lambda^{-1 / n}\right)$ suitably small so that

$$
J\left(X_{0}\right)=\inf \left\{J(X) ; \quad X \in \chi_{0}\right\} .
$$

For any $\varepsilon>0$, let $\varphi(s) \in \Phi$ so that $\sup _{s \in\left[\delta_{0}, \bar{s}\right]} J(\varphi(s)) \leq c_{0}+\varepsilon$. Let $X_{s}(\cdot, t)$ be the solution of (3.9). For any $s \in\left[\delta_{0}, \bar{s}\right]$, let

$$
t^{*}(s)=\sup \left\{t ; \quad X_{s}(\cdot, t) \in \chi_{0}\right\}
$$

Then $X_{s}\left(\cdot, t^{*}(s)\right) \in \partial \chi_{0}$, which implies

$$
J\left(X_{s}\left(\cdot, t^{*}(s)\right)\right) \geq \inf \left\{J(X), \quad X \in \chi_{0}\right\}=J\left(X_{0}\right) .
$$

Note that $R(X) \geq \frac{1}{2} \Lambda^{-1 / n}$ for any $X \in \chi_{0}$. By Remark 2.2 we have $R\left(X_{s}(\cdot, t)\right) \geq$ $\frac{1}{2} \Lambda^{-1 / n} \forall t \in\left(0, t^{*}(s)\right]$. 
By (3.6) we have

$$
\begin{aligned}
J\left(X_{s}\left(\cdot, t^{*}(s)\right)\right)-J\left(X_{s}(\cdot, 0)\right) & =\int_{0}^{t^{*}(s)} \frac{d}{d t} J\left(X_{s}(\cdot, t)\right) d t \\
& \leq-\int_{0}^{t^{*}(s)} \int_{S^{n}} \frac{\partial}{\partial t} H_{s}(x, t)\left\{e^{\frac{\partial}{\partial t} H_{s}}-1\right\} d \sigma d t,
\end{aligned}
$$

where $H_{s}(x, t)$ is the support function of $X_{s}(\cdot, t)$. By (iv) and (v) in the definition of $\Phi$ (see the proof of Theorem 3.1) we have $\left|\frac{\partial}{\partial t} H_{s}(x, t)\right| \leq \widetilde{C}_{1}=\widetilde{C}_{1}\left(A_{0}, B_{0}\right)$ for $t \in\left(0, t^{*}(s)\right)$. Hence if $\varphi(s) \in \chi_{0}$ for some $s$, there exists $C>0$ depends only on $\widetilde{C}_{1}$ and $\operatorname{dist}\left(\varphi(s), \partial \chi_{0}\right)$ so that

$$
t^{*}(s) \geq C
$$

Let

$$
s^{*}=\inf \left\{s ; t^{*}(s)=+\infty\right\} .
$$

For any $s<\frac{1}{2} \Lambda^{1 / 2}$, by Remark 2.2 we have $R\left(X_{s}(\cdot, t)\right)<R\left(X_{s}(\cdot, 0)\right)$. By (ii) of the definition of $\Phi$ in Theorem 3.1, $R\left(X_{s}(\cdot, 0)\right)=s<\frac{1}{2} \Lambda^{1 / 2}$, namely, $X_{s}(\cdot, t) \notin \chi_{0}$. Hence $s^{*}>\frac{1}{2} \Lambda^{1 / 2}>0$.

If $s^{*}=\bar{s}$, the left hand side of (3.17)

$$
\geq J\left(X_{0}\right)-J\left(X_{s}(\cdot, 0)\right)=J(\varphi(\bar{s}))-J(\varphi(s)) \rightarrow 0 \text { as } s \rightarrow \bar{s} .
$$

Hence by the a priori extimates in Section 2, and noticing that $t^{*}(s) \geq C>0$ for $s$ near $\bar{s}$, we have

$$
\frac{\partial}{\partial t} H_{s}(\cdot, t) \rightarrow 0 \text { uniformly for } t \in\left(0, t^{*}(s)\right) \text { as } s \rightarrow \bar{s} .
$$

Hence there is a subsequence $s_{j} \rightarrow \bar{s}$ so that $X_{s_{j}}\left(\cdot, t^{*}\left(s_{j}\right)\right)$ converges to a solution $X_{*}$ of $(1.1)$. Since $X_{s_{j}}\left(\cdot, t^{*}\left(s_{j}\right)\right) \in \partial \chi_{0}$, we have $X_{*} \in \partial \chi_{0}$.

Next we consider the case $s^{*}<\bar{s}$. Note that $X_{s^{*}}(\cdot, t)$ can not shrink to a point in finite time. By Lemma 3.4, there exists a subsequence of $X_{s^{*}}(\cdot, t)$ (still denoted by $\left.X_{s^{*}}(\cdot, t)\right)$ which converges to a solution $X_{*}$ of (1.1). If $X_{*} \neq X_{0}$, we are through. If $X_{*}=X_{0}$, we may choose $t_{j}>0$ so that $\operatorname{dist}\left(X_{0}, X_{s^{*}}\left(\cdot, t_{j}\right)\right)<2^{-j-1} \rho_{0}$. Hence there exists $s_{j} \in\left(\delta_{0}, s_{*}\right)$ so that

$$
\operatorname{dist}\left(X_{0}, X_{s_{j}}\left(\cdot, t_{j}\right)\right) \leq 2^{-j} \rho_{0},
$$

which implies $t^{*}\left(s_{j}\right)-t_{j} \geq C$ and

$$
J\left(X_{s_{j}}\left(\cdot, t_{j}\right)\right) \leq J\left(X_{0}\right)+\varepsilon_{j}
$$

with $\varepsilon_{j} \rightarrow 0$ as $j \rightarrow \infty$. Similar to (3.17) we have

$$
J\left(X_{s_{j}}\left(\cdot, t^{*}\left(s_{j}\right)\right)\right)-J\left(X_{s_{j}}\left(\cdot, t_{j}\right)\right) \leq-\int_{t_{j}}^{t^{*}\left(s_{j}\right)} \int_{S^{n}} \frac{\partial}{\partial t} H_{s_{j}}(x, t)\left\{e^{\frac{\partial}{\partial t} H_{s_{j}}}-1\right\} d \sigma d t,
$$

and the left hand side

$$
\geq J\left(X_{0}\right)-J\left(X_{s_{j}}\left(\cdot, t_{j}\right)\right) \geq-\varepsilon_{j} \rightarrow 0 \text { as } j \rightarrow \infty .
$$


Consequently

$$
\frac{\partial}{\partial t} H_{s_{j}}(\cdot, t) \rightarrow 0 \text { uniformly for } t \in\left(t_{j}, t^{*}\left(s_{j}\right)\right) \text { as } j \rightarrow \infty .
$$

Hence there exists a subsequence of $s_{j}$ (still denoted by $s_{j}$ ) so that $X_{s_{j}}\left(\cdot, t^{*}\left(s_{j}\right)\right.$ ) converges to a solution $X_{*}$ of (1.1) so that $X_{*} \in \partial \chi_{0}$. This completes the proof.

\section{Proof of Theorem 2}

In this section we suppose $f(x)$ satisfies (1.5) and $\|f(x)\|_{C^{2}\left(\mathbb{R}^{n+1}\right)} \leq C$. For any convex hypersurface $X_{0}$ with support center at $y$, let $X_{\theta}(\cdot, t)$ be the solution of the problem

$$
\frac{\partial}{\partial t} X=-\log (K / f) \cdot \gamma, \quad X(\cdot, 0)=y+\theta\left(X_{0}-y\right) .
$$

Suppose the maximal time interval of $X_{\theta}(\cdot, t)$ is $\left[0, T_{\theta}\right)$. By the comparison principle we have $X_{\theta_{1}}(\cdot, t)<X_{\theta_{2}}(\cdot, t)$ and $T_{\theta_{1}} \leq T_{\theta_{2}}$ for any $\theta_{1}<\theta_{2}$. And $T_{\theta}<\infty$ if $\theta$ is small enough, $R(X(\cdot, t)) \rightarrow+\infty$ if $\theta$ is large enough. Let $\chi(y)$ denote the set of convex hypersurfaces $X_{0}$ with support center at $y$, so that the solution $X_{\theta}(\cdot, t)$ shrinks to a point in finite time for $\theta \in(0,1)$, and $X_{\theta}(\cdot, t)_{\mid \theta=1}$ exists for all $t>0$, i.e., $T_{\theta}<+\infty$ for any $\theta<1$ and $\left.T_{\theta}\right|_{\theta=1}=\infty$.

Lemma 4.1. For $X_{0} \in \chi(y)$ we have

$$
R\left(X_{0}\right) \leq M_{0} \equiv \frac{n}{\omega_{n-1}}\left[J\left(X_{0}\right)+\omega_{n} \Lambda(4 / \lambda)^{(n+1) / n}\right] .
$$

Proof. By (3.2) we have

$$
J\left(X_{0}\right)=\int_{S^{n}} H_{0} d \sigma-\int_{C l\left(X_{0}\right)} f(x) d x \geq \frac{\omega_{n-1}}{n} R\left(X_{0}\right)-\Lambda \operatorname{Vol}\left(X_{0}\right) .
$$

Hence

$$
\operatorname{Vol}\left(X_{0}\right) \geq \frac{1}{\Lambda}\left[\frac{\omega_{n-1}}{n} R\left(X_{0}\right)-J\left(X_{0}\right)\right]
$$

Let $X_{\theta}(\cdot, t)$ be the solution of (4.1). By Lemma 3.5,

$$
\frac{d}{d t} \operatorname{Vol}\left(X_{\theta}(\cdot, t)\right) \geq \omega_{n}^{1 /(n+1)}\left[\operatorname{Vol}\left(X_{\theta}(\cdot, t)\right]^{n /(n+1)}-\frac{4}{\lambda} \omega_{n} .\right.
$$

If

$$
\operatorname{Vol}\left(X_{0}\right)>\omega_{n}\left(\frac{4}{\lambda}\right)^{(n+1) / n},
$$

then $\operatorname{Vol}\left(X_{\theta}(\cdot, 0)\right)>\omega_{n}(4 / \lambda)^{(n+1) / n}$ for $\theta<1$ sufficiently close to 1 . Hence $\operatorname{Vol}\left(X_{\theta}(\cdot, t)\right) \rightarrow+\infty$ for $\theta<1$ close to 1 . On the other hand, by definition we have $T_{\theta}<+\infty$ when $\theta<1$. This is a contradiction. Hence $\operatorname{Vol}\left(X_{0}\right) \leq \omega_{n}(4 / \lambda)^{(n+1) / n}$ and so (4.2) follows.

If $X_{0} \in \chi(y)$, we have $X_{\theta}\left(\cdot,\left.t\right|_{\left.\right|_{\theta=1}} \in \chi\left(y_{t}\right)\right.$ for any $t>0$, where $y_{t}$ is the support centre of $X_{\theta}(\cdot, t)_{\left.\right|_{\theta=1}}$. By (2.21), (4.2) and (3.6) we thus have

$$
\Lambda^{-1 / n} \leq R\left(\left.X_{\theta}(\cdot, t)\right|_{\theta=1} \leq \frac{n}{\omega_{n-1}}\left[J\left(X_{0}\right)+\omega_{n} \Lambda(4 / \lambda)^{(n+1) / n}\right] \quad \forall t \geq 0 .\right.
$$


For any convex hypersurface $X$, as in Section 3 we denote by $A(X)$ the supremum of the principal radii of $X$, by $B(X)$ we denote the supremum of the principal curvatures of $X$. Let

$$
\tilde{\chi}(y)=\left\{X_{0} \in \chi(y) ; \quad A\left(X_{0}\right) \leq A_{0}, B\left(X_{0}\right) \leq B_{0}\right\},
$$

and let $\tilde{\chi}=\bigcup\left\{\tilde{\chi}(y) ; y \in \mathbb{R}^{n+1}\right\}$. Set

$$
c(y)=c_{f}(y)=\inf \{J(X) ; \quad X \in \tilde{\chi}(y)\} .
$$

Similar to the proof of Theorem 3.1 we may take $A_{0}$ and $B_{0}$ large enough so that for any $X_{0} \in \tilde{\chi}$, the solution $X(\cdot, t)$ of

$$
\frac{\partial}{\partial t} X=-\log (K / f) \cdot \gamma, \quad X(\cdot, 0)=X_{0}
$$

satisfies

$$
A(X(\cdot, t)) \leq A_{0}, \quad B(X(\cdot, t)) \leq B_{0} \quad \forall t>0 .
$$

That is, for any $X_{0} \in \tilde{\chi}$, the solution of (4.8) belongs to $\tilde{\chi}$ for any $t>0$. Since the solution of (4.8) depends continuously on the initial surface $X_{0}$, we see that if $X_{i} \in \tilde{\chi}(y)$ and $X_{i} \rightarrow X_{0}$, then $X_{0} \in \tilde{\chi}(y)$. Hence $c(y)$ is attainable, namely, for any $y \in \mathbb{R}^{n+1}$, there exists $X_{y} \in \tilde{\chi}(y)$ so that $J\left(X_{y}\right)=c(y)$.

Lemma 4.2. $c(y)$ is Lipschitz continuous and there exists $c_{0}>0$ depending only on $n$ and $\Lambda$ so that

$$
c(y) \geq c_{0} \quad \forall y \in \mathbb{R}^{n+1} .
$$

Proof. For any $X_{0} \in \tilde{\chi}$, let $X_{\theta}(\cdot, t)$ be the solution of (4.1). Then for any $\theta \in(0,1)$, $X_{\theta}(\cdot, t)$ shrinks to a point in finite time. By $(3.6)$ and $(3.3)$,

$$
\begin{aligned}
& J\left(X_{1-\varepsilon}(\cdot, 0)\right)=\sup _{t>0} J\left(X_{1-\varepsilon}(\cdot, t)\right) \\
& \quad \geq \sup \left\{\frac{\omega_{n-1}}{n} R-\frac{\omega_{n} \Lambda}{n+1} R^{n+1}, 0<R<\Lambda^{-1 / n}\right\} \geq c_{0}=c_{0}(n, \Lambda) .
\end{aligned}
$$

Let $\varepsilon \rightarrow 0$, we obtain $J\left(X_{0}\right) \geq c_{0}>0$.

Next we prove that $c(y)$ is Lipschitz continuous. Let $X_{0} \in \widetilde{\chi}(y)$ so that $J\left(X_{0}\right)=$ $c(y)$. For $y^{\prime} \in \mathbb{R}^{n+1}$ near $y$ let $X_{0}^{\prime}=y^{\prime}-y+X_{0}$, and let $X_{\theta}^{\prime}(\cdot, t)$ be the solution of

$$
\frac{d}{d t} X=-\log (K / f) \cdot \gamma, \quad X(\cdot, 0)=y^{\prime}+\theta\left(X_{0}^{\prime}-y^{\prime}\right) .
$$

Let $\theta\left(y^{\prime}\right)=\sup \left\{\theta, \quad X_{\theta}^{\prime}(\cdot, t)\right.$ shrinks to a point in finite time $\}$. Let $\delta>0$ be the smallest constant so that $y^{\prime}+(1-\delta)\left(X_{0}^{\prime}-y^{\prime}\right) \subset X_{0} \subset y^{\prime}+(1+\delta)\left(X_{0}^{\prime}-y^{\prime}\right)$. Since $A\left(X_{0}\right) \leq A_{0}$ and $B\left(X_{0}\right) \leq B_{0}$, we have $\delta \leq C\left|y^{\prime}-y\right|$. By the comparison principle, $1-\delta \leq \theta\left(y^{\prime}\right) \leq 1+\delta$. We conclude $c\left(y^{\prime}\right) \leq c(y)+C_{1}\left|y-y^{\prime}\right|$ from the Lipschitz continuity of $f(x)$.

It is easy to see that $\forall y \in \mathbb{R}^{n+1}$,

$$
\begin{aligned}
c(y) & \leq \sup _{t>0} J\left(y+t S^{n}\right) \\
& \leq \sup _{t>0}\left\{\omega_{n} t-\frac{\lambda \omega_{n}}{n+1} t^{n+1}\right\} \\
& =n \omega_{n} /(n+1) \lambda^{1 / n} .
\end{aligned}
$$


We say $y_{0}$ is a local minimum of $c(y)$ if there exists a neighbourhood $N$ of $y_{0}$ so that $c\left(y_{0}\right)=\inf \{c(y) ; y \in N\}$.

Theorem 4.1. Suppose $c(y)$ attains its local minimum at $y_{0}$. Then (1.1) has a solution $X_{0} \in \tilde{\chi}\left(y_{0}\right)$ so that $J\left(X_{0}\right)=c\left(y_{0}\right)$.

Proof. Let $X_{0} \in \tilde{\chi}\left(y_{0}\right)$ so that $J\left(X_{0}\right)=c\left(y_{0}\right)$. If $X_{0}$ is not a solution of (1.1), we consider the solution $X(\cdot, t)$ of

$$
\frac{\partial}{\partial t} X=-\log K / f \cdot \gamma, \quad X(\cdot, 0)=X_{0}
$$

By (3.6) we have

$$
\frac{d}{d t} J(X(\cdot, t)) \leq-\int_{S^{n}} \frac{\partial}{\partial t} H(x, t)\left\{e^{\frac{\partial}{\partial t} H}-1\right\} d \sigma<0,
$$

where $H(x, t)$ is the support function of $X(\cdot, t)$. We obtain $c(p(X(\cdot, t))) \leq J(X(\cdot, t))$ $<J\left(X_{0}\right)=c\left(y_{0}\right)$ for any $t>0$. But for $t$ small the support centre $p(X(\cdot, t))$ lies in $N$ and hence by assumption we have $c(p(X(\cdot, t))) \geq c\left(y_{0}\right)$. This contradiction shows that $X_{0}$ is a solution of (1.1).

Corollary 4.1. Suppose there exists a domain $\Omega$ which contains $B_{M_{0}}(0)$ so that

$$
\inf \left\{f(x) ; \quad x \in B_{M_{0}}(0)\right\} \geq \sup \left\{f(x) ; \quad x \in N_{M_{0}}(\partial \Omega)\right\},
$$

where $N_{\delta}$ denotes the $\delta$ neighbourhood, and $M_{0}$ is the constant in Lemma 4.1 with $J\left(X_{0}\right) \leq n \omega_{n} /(n+1) \lambda^{1 / n}$ given by (4.11). Then (1.1) has at least one solution.

Proof. From (4.13) we have $c(0) \leq \inf \{c(y), y \in \partial \Omega\}$. Hence $c(y)$ attains its minimum in $\Omega$. By Theorem 4.1 we obtain a solution with support centre contained in $\Omega$.

We guess that if $c(y)$ attains its strict maximum at some point $y_{0}$, then (1.1) has a solution $X_{0} \in \tilde{\chi}\left(y_{0}\right)$. We will give an example to show that the constant $M_{0}$ in (4.13) cannot be too small.

Theorem 4.2. Suppose

$$
\lim _{|x| \rightarrow \infty} f(x)=f_{0}>0 .
$$

Then (1.1) has at least one solution. If furthermore $c(y)$ attains its maximum at some point, then (1.1) has a solution $X$ so that $J(X) \geq \sup \left\{c(y), y \in \mathbb{R}^{n+1}\right\}$.

Proof. We may suppose $f_{0}=1$. For any $X \in \widetilde{\chi}(y)$, by (4.5) we have

$$
J(X)=I_{n-1}(X)-\int_{C l(X)} d x+o(1),
$$

where $o(1) \rightarrow 0$ as $|p(X)| \rightarrow \infty$. Since $S^{n}$ is the unique solution of (1.1) with $f=1$, we have

$$
c(y)=\inf \{J(X), X \in \tilde{\chi}(y)\} \rightarrow \frac{n}{n+1} \omega_{n} \text { as }|y| \rightarrow \infty .
$$

If $\sup _{y \in \mathbb{R}^{n+1}} c(y)=\frac{n}{n+1} \omega_{n}$, then $c(y)$ attains its local minimum at some point and by Theorem 4.1 there is a solution of (1.1). Hence to prove Theorem 4.2 we may suppose $\sup _{y \in \mathbb{R}^{n+1}} c(y)>\frac{n}{n+1} \omega_{n}$ and the supremum is attained at the origin. 
For $y \in \mathbb{R}^{n+1}$, let $\theta(y)>0$ so that the solution $X_{\theta, y}(\cdot, t)$ of

$$
\frac{\partial}{\partial t} X=-\log (K / f) \cdot \gamma, \quad X(\cdot, 0)=\theta\left(y+S^{n}\right)
$$

shrinks to a point in finite time for $\theta \in(0, \theta(y))$, and $\left.X_{\theta, y}(\cdot, t)\right|_{\theta=\theta(y)}$ exists for all $t>0$. It is easy to see that $\theta(y)$ depends continuously on $y$ and $\theta(y) \rightarrow 1$ as $|y| \rightarrow \infty$. Let $d(y)=J\left(\theta(y)\left(y+S^{n}\right)\right)$. We have $d(y) \geq c(y)$, and $d(y) \rightarrow \frac{n}{n+1} \omega_{n}$ as $|y| \rightarrow \infty$. Hence there exists $R$ large enough so that $c(0)>d(y)$ for any $|y| \geq R / 2$.

Consider the solution $X_{y}(\cdot, t)=: X_{\theta(y), y}(\cdot, t)$. From $(3.6)$,

$$
J\left(X_{y}(\cdot, t)\right)-J\left(X_{y}(\cdot, 0)\right) \leq-\int_{0}^{t} \int_{S^{n}} \frac{\partial}{\partial t} H_{y}\left\{e^{\frac{\partial}{\partial t} H_{y}}-1\right\} d x d t,
$$

where $H_{y}(\cdot, t)$ is the support function of $X_{y}(\cdot, t)$. Since $X_{y}(\cdot, t) \in \tilde{\chi}$, by (4.9) the left hand side is uniformly bounded. By the a priori estimates in Section 2 we conclude that for any given $y \in \mathbb{R}^{n+1}$,

$$
\frac{\partial}{\partial t} H_{y}(x, t) \rightarrow 0 \quad \text { uniformly for } x \in S^{n} .
$$

Let $p_{y, t}$ be the support centre of $X_{y}(\cdot, t)$. We claim that for any $t \geq 0$, there exists $y_{t} \in B_{R}(0)$ so that $p_{y_{t}, t}=0$. Indeed,

$$
c\left(p_{y, t}\right) \leq J\left(X_{y}(\cdot, t)\right) \leq J\left(X_{y}(\cdot, 0)\right)<c(0)
$$

for any $|y| \geq R / 2$. Hence $p_{y, t} \neq 0$ for any $|y| \geq R / 2$. If the claim is not true at $t_{0}>0$, we introduce $G(y, s)=p_{y, t(y, s)}, s \in[0,1]$, where

$$
t(y, s)= \begin{cases}t_{0} s, & y \in B_{R / 2}(0), \\ t_{0} s\left(\frac{R}{|y|}-1\right), & y \in B_{R}(0) \backslash B_{R / 2}(0) .\end{cases}
$$

$G(y, s)$ is a homotopy from $B_{R}(0)$ to $\left\{G(y, 1), \quad y \in B_{R}(0)\right\}$ so that $G(y, s)=i d$ on $\partial B_{R}(0)$. Since $0 \notin\left\{G(y, 1), \quad y \in B_{R}(0)\right\}$, we obtain a continuous map $y \rightarrow$ $R \frac{G(y, 1)}{|G(y, 1)|}$ from $B_{R}(0)$ to $\partial B_{R}(0)$ which is the identity map on $\partial B_{R}(0)$. This is impossible.

Since $y_{t} \in B_{R}(0)$ we may suppose $y_{t} \rightarrow y_{0}$ by selecting a subsequence. We wish to show $p_{y_{0}, t}$ is uniformly bounded. Suppose on the contrary that there is a subsequence $t_{j} \rightarrow \infty$ so that $\left|p_{y_{0}, t_{j}}\right| \rightarrow \infty$. Since $f(y) \rightarrow 1$ as $|y| \rightarrow \infty$, by (4.16) we see that the Gauss curvature $K\left(X_{y_{0}}\left(\cdot, t_{j}\right)\right)$ of $X_{y_{0}}\left(\cdot, t_{j}\right)$ satisfies

$$
K\left(X_{y_{0}}\left(\cdot, t_{j}\right)\right)=f\left(X_{y_{0}}\left(\cdot, t_{j}\right)\right) \exp \left\{\frac{\partial}{\partial t} X_{y_{0}}\left(\cdot, t_{j}\right)\right\}=1+o(1)
$$

for $j$ large. By the uniqueness of solutions to the equation $K(X)=1$ we conclude

$$
X_{y_{0}}\left(\cdot, t_{j}\right) \subset N_{\delta_{j}}\left(S^{n}+p_{y_{0}, t_{j}}\right)
$$

with $\delta_{j} \rightarrow 0$ as $j \rightarrow \infty$. It follows

$$
J\left(X_{y_{0}}\left(\cdot, t_{j}\right)\right)=J\left(S^{n}+p_{y_{0}, t_{j}}\right)+o(1)=\frac{n}{n+1} \omega_{n}+o(1) .
$$

Hence there exists $j_{0}$ sufficiently large so that

$$
J\left(X_{y_{0}}\left(\cdot, t_{j_{0}}\right)\right)<c(0)
$$


which implies $J\left(X_{y}\left(\cdot, t_{j_{0}}\right)\right)<c(0)$ for $y$ sufficiently close to $y_{0}$. In particular for $t$ large enough we obtain

$$
J\left(X_{y_{t}}\left(\cdot, t_{j_{0}}\right)\right)<c(0) .
$$

Since $J\left(X_{y}(\cdot, t)\right)$ is nonincreasing, it follows

$$
J\left(X_{y_{t}}(\cdot, t)\right)<c(0) .
$$

On the other hand, since $p_{y_{t}, t}=0$ we have $J\left(X_{y_{t}}\left(\cdot, t_{j_{0}}\right)\right) \geq c\left(p_{y_{t}, t}\right)=c(0)$. We reach a contradiction. Hence $p_{y_{0}, t}$ is uniformly bounded.

By Lemma 3.4, we may select a subsequence $t_{j}$ so that $X_{y_{0}}\left(\cdot, t_{j}\right)$ converges to a solution $X_{0}$ of (1.1).

Finally we prove $J\left(X_{0}\right) \geq c(0)$. If this is not true, noticing that $J\left(X_{y_{0}}(\cdot, t)\right)$ is nonincreasing, we have

$$
\lim _{t \rightarrow \infty} J\left(X_{y_{0}}(\cdot, t)\right)=J\left(X_{0}\right)<c(0),
$$

namely, $J\left(X_{y_{0}}\left(\cdot, t_{0}\right)\right)<c(0)$ for some $t_{0}$ large enough. Similar to the above argument we obtain $J\left(X_{y_{t}}(\cdot, t)\right)<c(0)$ for $t$ large enough, which is in contradiction with $J\left(X_{y_{t}}(\cdot, t)\right) \geq c\left(p_{y_{t}, t}\right)=c(0)$. This completes the proof.

We give an example to show that the constant $M_{0}$ in Corollary 4.1 can not be too small. For simplicity we consider the case $n=1$. By the four vertex theorem we see that if $f(x)=3+\operatorname{arctg} x_{1}$, then there is no solution of (1.1).

Example 1. Let $f(x)=\lambda\left(3+\operatorname{arctg} x_{1} \pm g_{\varepsilon}(x)\right)$, where $\lambda \in(0,1]$ is a constant and

$$
g_{\varepsilon}(x)= \begin{cases}\cos \left(\pi \sqrt{x_{1}^{2}+x_{2}^{2}} / 2 \varepsilon\right) & \text { if } x_{1}^{2}+x_{2}^{2} \leq \varepsilon^{2}, \\ 0 & \text { else. }\end{cases}
$$

Then (1.1) has no solution if $\varepsilon$ is small.

Proof. Since

$$
\int_{X} f d s=\int_{X} K d \theta=2 \pi
$$

we have

$$
\frac{2}{\sup f} \leq R(X) \leq \frac{\pi}{\inf f} .
$$

If $X \cap B_{\varepsilon}(0)$ is empty, then $f(x)=\lambda\left(3+\operatorname{arctg} x_{1}\right)$ on $X$. By the four vertex theorem, there is no solution of (1.1). If $X \cap B_{\varepsilon}(0)$ is not empty, we have, by the definition of curvature,

$$
\operatorname{mes}\left(G\left(X \cap B_{\varepsilon}(0)\right)\right) \leq \sup f(x) \cdot \operatorname{mes}\left(X \cap B_{\varepsilon}(0)\right) \leq 12 \pi \lambda \varepsilon,
$$

where $G: X \rightarrow S^{1}$ is the Gauss map of $X$. We compute

$$
\int_{S^{1}} \frac{x_{1}}{f\left(G^{-1}(x)\right)} d s=\int_{0}^{2 \pi} \frac{\sin \theta}{f(x(\theta))} d \theta=\int_{0}^{\pi}\left(\frac{\sin \theta}{f(x(\theta))}-\frac{\sin \theta}{f(x(2 \pi-\theta))}\right) d \theta,
$$


where $\theta$ is the angle between $o x$ and the minus $x_{2}$ direction, $x(\theta)=G^{-1}(\sin \theta,-\cos \theta)$. We have

$$
\begin{aligned}
\int_{S^{1}} \frac{x_{1}}{f} d s & \leq \int_{0}^{\pi}\left(\frac{\sin \theta}{f_{0}(x(\theta))}-\frac{\sin \theta}{f_{0}(x(2 \pi-\theta))}\right) d \theta+\frac{2}{\inf f} \cdot \operatorname{mes}\left(G\left(X \cap B_{\varepsilon}(0)\right)\right) \\
& \leq \int_{0}^{\pi}\left(\frac{\sin \theta}{f_{0}(x(\theta))}-\frac{\sin \theta}{f_{0}(x(2 \pi-\theta))}\right) d \theta+\frac{48 \pi \varepsilon}{4-\pi}
\end{aligned}
$$

where $f_{0}=\lambda\left(3+\operatorname{arctg} x_{1}\right)$. By Lemma 3.1 and (4.18),

$$
r(X) \geq R(X)^{2} / 8 \sup (1 / f(x)) \geq \frac{1}{2} \inf f(x) /|\sup f(x)|^{2} .
$$

Hence $x_{1}(\theta)-x_{1}(2 \pi-\theta) \geq \sin \theta r(X) \geq C \sin \theta / \lambda$. We have

$$
\frac{1}{f_{0}(x(\theta))}-\frac{1}{f_{0}(2 \pi-\theta)} \leq-[\sup f]^{-2}[f(x(\theta))-f(x(2 \pi-\theta))] \leq-\frac{C}{\lambda} \sin \theta
$$

for some $C>0$ independent of $\lambda \in(0,1]$. We thus obtain

$$
\int_{S^{1}} \frac{x_{1}}{f} d s \leq-\frac{C}{\lambda}+\frac{48 \pi \varepsilon}{4-\pi} .
$$

Hence if $\varepsilon \leq C_{1} / \lambda$ for some $C_{1}>0$, then $\int_{S^{1}} \frac{x_{1}}{f} d s<0$. But on the other hand, by the necessary condition for the Minkowski problem, we have $\int_{S^{1}} \frac{x_{1}}{f} d s=0$, a contradiction.

\section{REFERENCES}

1. Yu. D. Burago and V. A. Zalgaller, Geometric Inequalities, Springer-Verlag, 1980. MR 82d:52009

2. K. C. Chang, Infinite dimensional Morse theory and multiple solution problems, Boston, 1993. MR 94e: 58023

3. S. Y. Cheng and S. T. Yau, On the regularity of the solution of the n-dimensional Minkowski problem, Comm. Pure Appl. Math. 29 (1976), 495-516. MR 54:11247

4. K. S. Chou and X. J. Wang, The logarithmic Gauss curvature flow, preprint.

5. H. Delanoë, Plongements radiaux $S^{n} \rightarrow \mathbb{R}^{n+1}$ a courbure de Gauss positive prescrite, Ann. Sci. Ècole Norm. Sup. (4) 18 (1986), 635-649. MR 87j:53011

6. V. I. Oliker, Hypersurfaces in $\mathbb{R}^{n+1}$ with prescribed Gaussian curvature and related equations of Monge-Ampère type, Comm. Partial Diff. Eqns. 9 (1984), 807-837. MR 85h:53047

7. V. I. Oliker, The problem of embedding $S^{n}$ into $\mathbb{R}^{n+1}$ with prescribed Gauss curvature and its solution by variational methods, Trans. Amer. Math. Soc. 295 (1986), 291-303. MR 87i:53092

8. A. Pogorelov, The Minkowski multidimensional problem, J. Wiley, New York, 1978. MR $\mathbf{5 7 : 1 7 5 7 2}$

9. R. C. Reilly, Variational properties of functions of the mean curvatures for hypersurfaces in space forms, J. Diff. Geom. 9 (1973), 465-477. MR 49:6102

10. K. S. Tso, Convex hypersurfaces with prescribed Gauss-Kronecker curvature, J. Diff. Geom. 34 (1991), 389-410. MR 92j:53029

11. K. S. Tso, On a Monge-Ampère functional, Invent. Math. 101 (1990), 425-448. MR 91i:35082

12. K. S. Tso, Deforming a hypersurface by its Gauss-Kronecker curvature, Comm. Pure Appl. Math. 38 (1985), 867-882. MR 87c:53009

13. S. T. Yau, Problem section, Seminar on differential geometry, Ann. of Math. Studies No. 102, Princeton Univ. Press, 1982, pp. 669-706. MR 83e:53029

Department of Mathematics, Zhejiang University, Hangzhou 310027, P.R. China Current address: School of Mathematical Sciences, Australian National University, Canberra, ACT 0200, Australia

E-mail address: wang@pell.anu.edu.au 